\title{
过渡金属修饰 $\left\{\mathbf{P}_{2} \mathbf{W}_{12}\right\}$ 基多酸化合物的研究进展
}

\author{
辛兴, 吕红金* \\ 北京理工大学化学与化工学院, 原子分子簇科学教育部重点实验室, 北京 102488 \\ *通讯作者, E-mail: hlv@bit.edu.cn \\ 收稿日期：2020-05-14; 接受日期：2020-05-25; 网络版发表日期：2020-07-09 \\ 国家自然科学基金(编号: 21871025, 21831001)资助项目
}

摘要多金属氧酸盐由于具有独特的结构组成与物理化学性质, 如原子结构明晰、组成多样可控、得失电子/ 质子稳定可逆、丰富的氧化还原特性等, 目前已成为无机化学中历史较为悠久、交叉渗透最广的研究领域之一. 本文综述了多金属氧酸盐领域的重要系列之一——过渡金属修饰 $\left\{\mathrm{P}_{2} \mathrm{~W}_{12}\right\}$ 基多酸化合物的研究进展, 根据 $\left\{\mathrm{P}_{2} \mathrm{~W}_{12}\right\}$ 构筑模块的聚合数分类归纳了该系列化合物的相关结构, 阐述了部分代表性化合物的结构特点及合成策 略, 整理了该类化合物的功能应用, 并对其未来发展趋势进行了展望.

关键词多金属氧酸盐, 过渡金属修饰, $\left\{\mathrm{P}_{2} \mathrm{~W}_{12}\right\}$ 和 $\left\{\mathrm{P}_{8} \mathrm{~W}_{48}\right\}$ 构筑模块

\section{1 引言}

多金属氧酸盐(又称多酸或多金属氧簇, polyoxometalates，POMs)是一类由前过渡元素Mo、W、V、 $\mathrm{Nb}$ 和 $\mathrm{Ta}$ 通过氧连接形成的具有固定化学组成和几何 结构的金属-氧簇合物(metal-oxo clusters), 它们独特的 结构与物理化学性质使其在催化 ${ }^{[1 \sim 4]}$ 、磁性 ${ }^{[5 \sim 7]}$ 、电化 学 ${ }^{[8 \sim 10]}$ 、医药 ${ }^{[11 ~ 13]}$ 、材料科学 ${ }^{[14 \sim 16]}$ 等领域有着广泛的 应用. 截至目前, 多酸化学领域已有近两百年的历史. 随着近代各类谱学表征手段、分析方法、理论计算以 及X射线结晶学的快速发展, 研究者对该领域的认识 与研究逐步加深, 各种与多酸化学交叉的研究领域层 出不穷, 表明了该领域的发展活力与广阔前景 ${ }^{[17 ~ 20]}$.

$\mathrm{POMs}$ 的基本构筑单元是 $\left\{\mathrm{MO}_{6}\right\}$ 八面体和 $\left\{\mathrm{XO}_{4}\right\}$ 中心杂原子四面体，这些多面体通过共边、共角或共 面连接形成不同类型的多酸阴离子结构, 主要包括
Keggin、Wells-Dawson、Anderson、 Lindqvist、Dexter-Silverton、Allman-Waugh、Strandberg和WeakleyYamase等经典结构 ${ }^{[9,18,21,22]}$. 其中, 饱和型Keggin或 Wells-Dawson结构在合适条件下能够可控地移除一个或 者多个 $[\mathrm{M}=\mathrm{O}]^{4+}$ 单元，从而得到系列具有不同缺位位点 (lacunary site)的多酸配体, 如 $\left\{\mathrm{XW}_{11} \mathrm{O}_{39}\right\} 、\left\{\mathrm{XW}_{10} \mathrm{O}_{36}\right\}$ 、 $\left\{\mathrm{XW}_{9} \mathrm{O}_{34}\right\} 、\left\{\mathrm{X}_{2} \mathrm{~W}_{15} \mathrm{O}_{56}\right\} 、\left\{\mathrm{X}_{2} \mathrm{~W}_{12} \mathrm{O}_{48}\right\}$ 和 $\left\{\mathrm{X}_{8} \mathrm{~W}_{48} \mathrm{O}_{184}\right\}$ $(X=S i 、 P 、 A s 、 G e \text { 等元素 })^{[23 \sim 25]}$. 缺位型无机多酸配 体具有丰富的氧配位位点, 能够非常容易地和 $d 、 f 区$ 过渡金属离子发生配位反应从而形成过渡金属取代的 $\mathrm{POMs}^{[26 ~ 28]}$. 使用缺位多酸构筑模块制备纳米尺度的 过渡金属取代多酸化合物已发展成为非常成熟的合成 策略. 在该策略的实施中, 使用多缺位POMs构筑模块 通常更利于诱导形成高核数过渡金属团簇 ${ }^{[24]}$, 而过渡 金属离子的高核化有助于衍生出金属原子/单金属酸 根 $\left(\mathrm{MO}_{x}{ }^{n-}\right)$ 所不具备的新䓉物化性能 ${ }^{[29,30]}$.

引用格式: Xin X, Lv H. Advances of transition-metal-modified $\left\{\mathrm{P}_{2} \mathrm{~W}_{12}\right\}$-based polyoxometalates. Sci Sin Chim, 2020, 50: 1015-1030, doi: 10.1360/SSC-20200075 
在上述缺位多酸构筑模块中, $\left\{\mathrm{P}_{2} \mathrm{~W}_{12} \mathrm{O}_{48}\right\}$ (简称 $\left.\left\{\mathrm{P}_{2} \mathrm{~W}_{12}\right\}\right)$ 是由Wells-Dawson型 $\left[\mathrm{P}_{2} \mathrm{~W}_{18} \mathrm{O}_{62}\right]^{6-}$ 分解生成的 一种六缺位阴离子簇; 在适当合成条件下, $\left\{\mathrm{P}_{2} \mathrm{~W}_{12}\right\}$ 可 以产生不同程度的聚合体，形成具有更大体积的 $\left\{\mathrm{P}_{4} \mathrm{~W}_{24}\right\}$ 二聚体、 $\left\{\mathrm{P}_{6} \mathrm{~W}_{36}\right\}$ 三聚体或 $\left\{\mathrm{P}_{8} \mathrm{~W}_{48}\right\}$ 四聚体, 多 聚体空腔内的多个缺位能够与过渡金属/稀土金属离 子进行配位, 以形成具有多过渡金属取代的 $\left\{\mathrm{P}_{2} \mathrm{~W}_{12}\right\}$ 多 聚体(图1).

使用 $\left\{\mathrm{P}_{2} \mathrm{~W}_{12}\right\}$ 构筑模块合成多酸化合物的报道最 早出现于1997年. 在Hill研究组 ${ }^{[31]}$ 的报道中, $\left\{\mathrm{P}_{2} \mathrm{~W}_{12}\right\}$ 可与Linqvist型 $\left\{\mathrm{Nb}_{6}\right\}$ 聚阴离子在含有 $\mathrm{H}_{2} \mathrm{O}_{2}$ 的 $\mathrm{HCl} / \mathrm{LiCl}$ 溶液中反应，通过补位填充形成化合物 $\left(\mathrm{H}_{3} \mathrm{Cs}_{5.6} \mathrm{~K}_{3.1}\right.$ $\left.\mathrm{Li}_{0.3}\right)\left[\mathrm{P}_{2} \mathrm{~W}_{12}\left(\mathrm{NbO}_{2}\right)_{6} \mathrm{O}_{56}\right] \cdot 11 \mathrm{H}_{2} \mathrm{O}\left(\left\{\mathbf{N b}_{6}\right\}\right.$ ，图2(a)). 尽管 $\mathrm{H}_{2} \mathrm{O}_{2}$ 的加入使其稳定性有所增加，但该化合物在溶液 和固态下的稳定性都较差，因此具有相同构型的聚过 氧铇酸盐和聚过氧钼酸盐均未能被顺利地合成与表 征. 这方面的相关报道自2004年后逐渐增多，国内外主 要研究者包括王恩波、苏忠民、牛景杨、Achim Müller、Leroy Cronin、Ulrich Kortz、Paul Kögerler、 Louis Nadjo和Pierre Gouzerh等. 经过多年发展, 大量以 $\left\{\mathrm{P}_{2} \mathrm{~W}_{12}\right\}$ 为构筑模块的多酸化合物被合成和报道, 例如 $\left\{\mathrm{P}_{2} \mathrm{~W}_{12}\right\}$ 单体基 $\left\{\mathbf{F e}_{9}\right\}$ 和 $\left\{\mathbf{M}_{2}\right\}(\mathrm{M}=\mathrm{Mn} 、 \mathrm{Co} 、 \mathrm{Ni} 、 \mathrm{Cu}$ 或

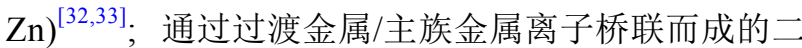
聚体基 $\left\{\mathrm{Fe}_{8}\right\} 、\left\{\mathrm{Fe}_{8} \mathrm{Co}_{2}\right\} 、\left\{\mathrm{Co}_{2} \mathrm{~W}_{2}\right\} 、\left\{\mathrm{Co}_{3}(\mathrm{PhPO})_{2}\right\}$ 、

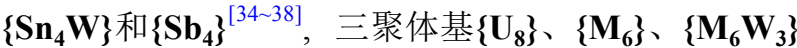

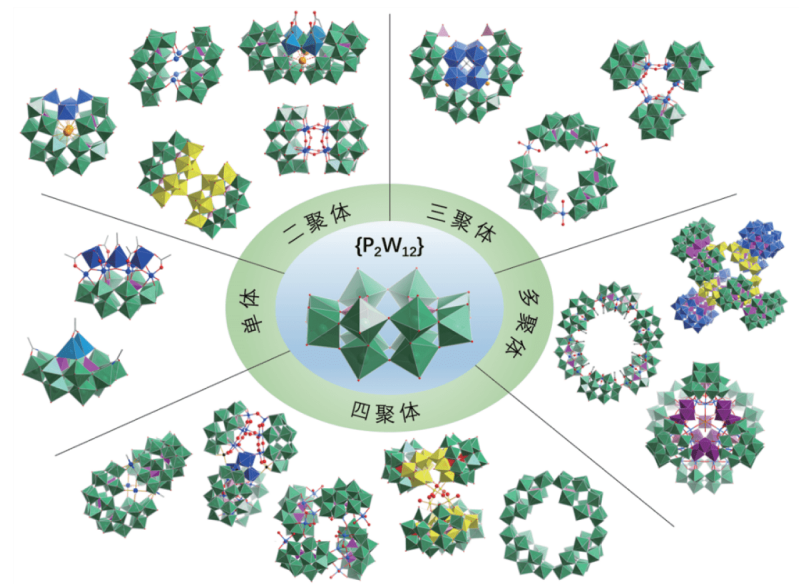

图 1 基于 $\left\{\mathrm{P}_{2} \mathrm{~W}_{12}\right\}$ 单体/多聚体的多酸化合物汇总图(网络 版彩图)

Figure 1 Summary of POM compounds based on $\left\{\mathrm{P}_{2} \mathrm{~W}_{12}\right\}$ monomer/ multimers (color online)
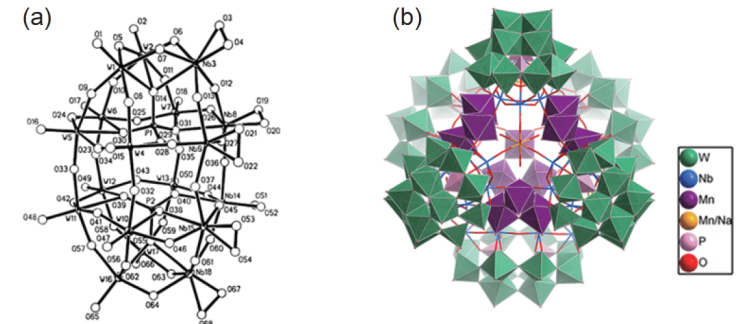

(c)

$\left[\left\{\mathrm{P}_{2} \mathrm{~W}_{12} \mathrm{Nb}_{7} \mathrm{O}_{63}\left(\mathrm{H}_{2} \mathrm{O}\right)_{2} \mathrm{KNb}_{4} \mathrm{O}_{4}(\mathrm{OH})_{6}\right\}\right]^{30}-\left\{\mathrm{Nb}_{32}\right\}$

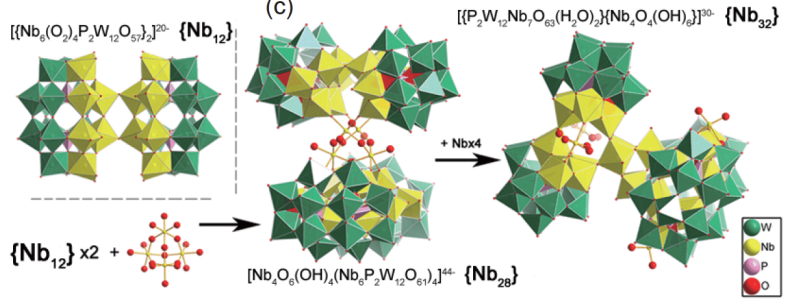

图 2 (a) 化合物 $\left\{\mathbf{N b}_{6}\right\}$ 聚阴离子的球棍示意图; (b) $\left\{\mathbf{M n}_{15}\right\}$ 聚 阴离子的结构示意图; (c) $\left\{\mathbf{N b}_{12}\right\} 、\left\{\mathbf{N b}_{28}\right\}$ 和 $\left\{\mathbf{N b}_{32}\right\}$ 聚阴离子 的结构示意图, 及三者间的转化关系(网络版彩图)

Figure 2 The ball-and-stick and polyhedral illustration of (a) $\left\{\mathbf{N b}_{6}\right\}$ polyanion, (b) $\left\{\mathbf{M n}_{15}\right\}$ polyanion, and (c) $\left\{\mathbf{N b}_{12}\right\},\left\{\mathbf{N b}_{28}\right\}$, and $\left\{\mathbf{N b}_{32}\right\}$ polyanions as well as the transformation between them (color online).

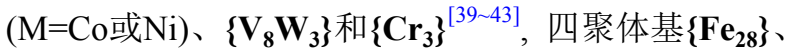
$\left\{\mathbf{S n}_{4}\right\}$ 和 $\left\{\mathbf{M n}_{16}\right\}^{[32,44,45]}$ ，六聚体 $\left\{\mathbf{M n}_{15}\right\} 、\left\{\mathbf{M n}_{24}\right\}^{[45,46]}$ 和 以 $\left\{\mathrm{P}_{8} \mathrm{~W}_{48}\right\}$ 为构筑模块的 $\left\{\mathbf{C u}_{20}\right\} 、\left\{\mathbf{C u}_{20}\right.$-azido $\}$ 、 $\left\{\mathrm{Cu}_{10}\right\} 、\left\{\mathrm{~V}_{12}\right\} 、\left\{\mathrm{Mo}_{12}\right\} 、\left\{\mathrm{Mo}_{8} \mathrm{~S}_{8}\right\},\left\{\mathrm{Mn}_{8}\right\} 、\left\{\mathrm{Mn}_{14}\right\} 、$ $\left\{\mathbf{M n}_{40}\right\} 、\left\{\mathrm{Fe}_{16}\right\} 、\left\{\mathrm{Co}_{10}\right\} 、\left\{\mathrm{Al}_{16}\right\} 、\left\{\mathrm{Se}_{4}\right\} 、\left\{(\mathrm{PhAs})_{4}\right\}$ 等

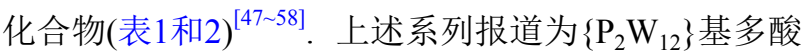
化合物的合成积累了大量经验，本文综述了该系列化 合物的研究进展，希望通过对其归纳整理分析，以阐 述其合成思路、结构特点、独特优势和功能应用，并 对其未来发展趋势进行了展望; 其中 $\left\{\mathrm{P}_{2} \mathrm{~W}_{12}\right\}$ 单体及 多聚体基多酸化合物归纳总结于表 $1,\left\{\mathrm{P}_{8} \mathrm{~W}_{48}\right\}$ 基多酸 化合物归纳总结于表2.

\section{$2\left\{\mathbf{P}_{2} \mathbf{W}_{12}\right\}$ 基多酸化合物的合成和结构}

\section{$2.1\left\{\mathbf{P}_{2} \mathbf{W}_{12}\right\}$ 单体基多酸化合物}

尽管 $\mathrm{Nb}$ 修饰的 $\left\{\mathrm{P}_{2} \mathrm{~W}_{12}\right\}$ 早在1997年就被合成和表 征，后续的系列研究直到2015年才被牛景杨团队报道. 在该系列工作中, 四例多聚阴离子簇: $\left\{\mathrm{Mn}_{15}\left(\mathrm{Nb}_{6} \mathrm{P}_{2} \mathrm{~W}_{12}\right.\right.$ $\left.\left.\mathrm{O}_{62}\right)_{6}\right\}^{n-} 、\left[\left\{\mathrm{Nb}_{6}\left(\mathrm{O}_{2}\right)_{4} \mathrm{P}_{2} \mathrm{~W}_{12} \mathrm{O}_{57}\right\}_{2}\right]^{20-} 、\left[\left\{\mathrm{Nb}_{4} \mathrm{O}_{6}(\mathrm{OH})_{4}\right\}\right.$ $\left.\left\{\mathrm{Nb}_{6} \mathrm{P}_{2} \mathrm{~W}_{12} \mathrm{O}_{61}\right\}_{4}\right]^{36-}$ 和 $\left[\mathrm{P}_{2} \mathrm{~W}_{12} \mathrm{Nb}_{7} \mathrm{O}_{63}\left(\mathrm{H}_{2} \mathrm{O}\right)_{2}\right\}_{4}\left\{\mathrm{Nb}_{4} \mathrm{O}_{4}\right.$ $\left.\left.(\mathrm{OH})_{6}\right\}\right]^{30-}$ 被相继合成 ${ }^{[46,59 \sim 61]}$. 根据结构中的杂原子修 饰数量, 可将其分别简称为 $\left\{\mathbf{M n}_{15}\right\} 、\left\{\mathbf{N b}_{12}\right\} 、\left\{\mathbf{N b}_{28}\right\}$ 和 
表 1 不同金属修饰的 $\left\{\mathbf{P}_{\mathbf{2}} \mathbf{W}_{12}\right\}$ 基多酸化合物

Table 1 Different metal-modified $\left\{\mathbf{P}_{\mathbf{2}} \mathbf{W}_{12}\right\}$-based polyoxometalates

\begin{tabular}{|c|c|c|c|}
\hline 阴离子簇的分子式 & 简称 & 特殊合成条件(原料/ $\mathrm{pH} /$ 加热 $)^{\mathrm{a})}$ & 文献 \\
\hline$\left[\mathrm{P}_{2} \mathrm{~W}_{12}\left(\mathrm{NbO}_{2}\right)_{6} \mathrm{O}_{56}\right]^{12-}$ & $\left\{\mathrm{Nb}_{6}\right\}$ & $\left\{\mathrm{Nb}_{6} \mathrm{O}_{19}\right\}+\mathrm{H}_{2} \mathrm{O}_{2}, \mathrm{pH} 0.85$ & {$[31]$} \\
\hline$\left[\left\{\mathrm{Nb}_{6}\left(\mathrm{O}_{2}\right)_{4} \mathrm{P}_{2} \mathrm{~W}_{12} \mathrm{O}_{57}\right\}_{2}\right]^{20-}$ & $\left\{\mathbf{N b}_{12}\right\}$ & $\left\{\mathrm{Nb}_{6} \mathrm{O}_{19}\right\}+\mathrm{H}_{2} \mathrm{O}_{2}, \mathrm{pH} 2,70^{\circ} \mathrm{C}(6 \mathrm{~h})$ & {$[60]$} \\
\hline$\left[\mathrm{H}_{123} \mathrm{Nb}_{36} \mathrm{P}_{12} \mathrm{~W}_{72} \mathrm{Mn}_{12}^{\mathrm{III}} \mathrm{Mn}_{3}{ }_{3}^{\mathrm{II}} \mathrm{NaO}_{424}\right]^{10-}$ & $\left\{\mathbf{M n}_{15}\right\}$ & $\left\{\mathrm{Nb}_{6} \mathrm{O}_{19}\right\}+\mathrm{H}_{2} \mathrm{O}_{2}, \mathrm{pH} 2.4,90^{\circ} \mathrm{C}(10 \mathrm{~h})$ & {$[46]$} \\
\hline$\left[\left\{\mathrm{Nb}_{4} \mathrm{O}_{6}(\mathrm{OH})_{4}\right\}\left\{\mathrm{Nb}_{6} \mathrm{P}_{2} \mathrm{~W}_{12} \mathrm{O}_{61}\right\}_{4}\right]^{36-}$ & $\left\{\mathbf{N b}_{28}\right\}$ & $\left\{\mathrm{Nb}_{6} \mathrm{O}_{19}\right\}+\mathrm{H}_{2} \mathrm{O}_{2}, \mathrm{pH} 1.7,80^{\circ} \mathrm{C}$ & [59] \\
\hline$\left[\left\{\mathrm{P}_{2} \mathrm{~W}_{12} \mathrm{Nb}_{7} \mathrm{O}_{63}\left(\mathrm{H}_{2} \mathrm{O}\right)_{2}\right\}_{4}\left\{\mathrm{Nb}_{4} \mathrm{O}_{4}(\mathrm{OH})_{6}\right\}\right]^{30-}$ & $\left\{\mathbf{N b}_{32}\right\}$ & $\left\{\mathrm{Nb}_{6} \mathrm{O}_{19}\right\}+\mathrm{H}_{2} \mathrm{O}_{2}, \mathrm{pH} 1.7,80^{\circ} \mathrm{C}$ & {$[60]$} \\
\hline$\left[\mathrm{H}_{4} \mathrm{P}_{2} \mathrm{~W}_{12} \mathrm{Fe}_{9} \mathrm{O}_{56}(\mathrm{OAc})_{7}\right]^{6-}$ & $\left\{\mathrm{Fe}_{9}\right\}$ & pH 3.7, 常温 & {$[32]$} \\
\hline$\left[\gamma-\mathrm{P}_{2} \mathrm{~W}_{12} \mathrm{O}_{44} \mathrm{M}_{2}(\mathrm{OAc})\left(\mathrm{CH}_{3} \mathrm{CONH}\right)_{2}\right]^{5-}$ & $\left\{M_{2}\right\}^{\text {b) }}$ & $\mathrm{TBABr}$, 于 $45^{\circ} \mathrm{C}$ 乙腈中重结晶 & {$[33]$} \\
\hline$\left[\mathrm{H}_{12} \mathrm{Fe}_{8} \mathrm{P}_{4} \mathrm{~W}_{28} \mathrm{O}_{120}\right]^{16-}$ & $\left\{\mathbf{F e}_{8}\right\}$ & $95^{\circ} \mathrm{C}(1 \mathrm{~h})$ & {$[34]$} \\
\hline$\left[\left\{\mathrm{M}\left(\mathrm{H}_{2} \mathrm{O}\right)_{4}\right\}_{2}\left\{\mathrm{H}_{12} \mathrm{Fe}_{8} \mathrm{P}_{4} \mathrm{~W}_{28} \mathrm{O}_{120}\right\}\right]^{12-}$ & $\left\{\mathrm{Fe}_{8} \mathrm{M}_{2}\right\}^{\mathrm{c})}$ & {$\left[\mathrm{Fe}_{2}^{\mathrm{III}}{ }_{2} \mathrm{M}^{\mathrm{II}} \mathrm{O}(\mathrm{OAc})_{6}\left(\mathrm{H}_{2} \mathrm{O}\right)_{3}\right], 95^{\circ} \mathrm{C}(2 \mathrm{~h})$} & {$[34]$} \\
\hline$\left[\mathrm{Mn}\left(\mathrm{H}_{2} \mathrm{O}\right)_{2}\left(\mathrm{~W}_{4} \mathrm{Mn}_{4} \mathrm{O}_{12}\right)\left(\mathrm{P}_{2} \mathrm{~W}_{14} \mathrm{O}_{54}\right)_{2}\right]^{18-}$ & $\left\{\mathbf{M n}_{5} \mathbf{W}_{4}\right\}$ & $\mathrm{enMe}+$ 均苯四甲酸二酐, $140^{\circ} \mathrm{C}(4 \mathrm{~d})$ & {$[64]$} \\
\hline$\left[\left\{\mathrm{W}_{2} \mathrm{Co}_{2} \mathrm{O}_{8}\left(\mathrm{H}_{2} \mathrm{O}\right)_{2}\right\}\left(\mathrm{P}_{2} \mathrm{~W}_{12} \mathrm{O}_{46}\right)_{2}\right]^{20-}$ & $\left\{\mathrm{Co}_{2} \mathrm{~W}_{2}\right\}$ & pH 3, 煮沸(10 h) & {$[65]$} \\
\hline$\left[\left(\mathrm{C}_{6} \mathrm{H}_{5} \mathrm{PO}\right)_{2} \mathrm{P}_{4} \mathrm{~W}_{24} \mathrm{O}_{92}\right]^{16-}$ & $\left\{(\mathrm{PhPO})_{2}\right\}$ & $\mathrm{PhPO}_{3} \mathrm{H}_{2}+\left[\left(\mathrm{CH}_{3}\right)_{2} \mathrm{NH}_{2}\right] \mathrm{Cl}, \mathrm{pH} 3,60^{\circ} \mathrm{C}(1 \mathrm{~d})$ & {$[36]$} \\
\hline$\left\{\left[\mathrm{Co}\left(\mathrm{H}_{2} \mathrm{O}\right)_{4}\right]_{2}\left[\left\{\left(\mathrm{H}_{2} \mathrm{O}\right)_{4} \mathrm{Co}\right\}(\mathrm{PhPO})_{2} \mathrm{P}_{4} \mathrm{~W}_{24} \mathrm{O}_{92}\right]\right\}^{10-}$ & $\left\{\mathrm{Co}_{3}(\mathrm{PhPO})_{2}\right\}$ & $\mathrm{PhPO}_{3} \mathrm{H}_{2}+\left[\left(\mathrm{CH}_{3}\right)_{2} \mathrm{NH}_{2}\right] \mathrm{Cl}, \mathrm{pH} 3,60^{\circ} \mathrm{C}(2 \mathrm{~d})$ & {$[36]$} \\
\hline$\left[\mathrm{H}_{6}\left\{\mathrm{P}_{2} \mathrm{~W}_{13} \mathrm{O}_{51}\right\}_{2} \mathrm{Sb}_{4}\right]^{10-}$ & $\left\{\mathbf{S b}_{4}\right\}$ & $\mathrm{C}_{4} \mathrm{H}_{4} \mathrm{KO}_{7} \mathrm{Sb}, \mathrm{pH} 3.8,75^{\circ} \mathrm{C}(45 \mathrm{~min})$ & {$[38]$} \\
\hline$\left[\left\{\mathrm{Sn}\left(\mathrm{CH}_{2}\right)_{2} \mathrm{COO}\right\}_{4}\left(\mathrm{H}_{2} \mathrm{O}\right)_{2}\left\{\mathrm{WO}_{5}\left(\mathrm{H}_{2} \mathrm{O}\right)\right\}\left(\mathrm{P}_{2} \mathrm{~W}_{15} \mathrm{O}_{56}\right)_{2}\right]^{20-}$ & $\left\{\mathbf{S n}_{4} \mathbf{W}\right\}$ & $\mathrm{Cl}_{3} \mathrm{Sn}\left(\mathrm{CH}_{2}\right)_{2} \mathrm{COOCH}_{3}, \mathrm{pH} 4,75^{\circ} \mathrm{C}(2 \mathrm{~h})$ & {$[37]$} \\
\hline$\left[\mathrm{Li}\left(\mathrm{H}_{2} \mathrm{O}\right) \mathrm{K}_{4}\left(\mathrm{H}_{2} \mathrm{O}\right)_{3}\left\{\left(\mathrm{UO}_{2}\right)_{4}\left(\mathrm{O}_{2}\right)_{4}\left(\mathrm{H}_{2} \mathrm{O}\right)_{2}\right\}_{2}\left(\mathrm{PO}_{3} \mathrm{OH}\right)_{2} \mathrm{P}_{6} \mathrm{~W}_{36} \mathrm{O}_{136}\right]^{25-}$ & $\left\{\mathbf{U}_{8}\right\}$ & $\mathrm{UO}_{2}\left(\mathrm{NO}_{3}\right)_{2}+\mathrm{H}_{2} \mathrm{O}_{2}, \mathrm{pH} 4,50^{\circ} \mathrm{C}(1 \mathrm{~h})$ & [39] \\
\hline$\left[\left(\mathrm{Mn}_{2}^{\mathrm{III}} \mathrm{P}_{2} \mathrm{~W}_{16} \mathrm{O}_{60}\right)_{3}\right]^{24-}$ & $\left\{\mathbf{M n}_{6}\right\}$ & $\left(\mathrm{NH}_{4}\right)_{2}\left[\mathrm{Ce}\left(\mathrm{NO}_{3}\right)_{6}\right], \mathrm{pH} 4$, 常温 & {$[66]$} \\
\hline$\left[\mathrm{K}_{2}\left(\mathrm{Fe}_{2}^{\mathrm{II}} \mathrm{P}_{2} \mathrm{~W}_{16} \mathrm{O}_{60}\right)_{3}\right]^{25-}$ & $\left\{\mathrm{Fe}_{6}\right\}$ & $\left\{\mathrm{P}_{2} \mathrm{~W}_{18}\right\}+$ Tris, pH 7, 常温 & {$[41]$} \\
\hline$\left[\mathrm{K}\left(\mathrm{Co}_{2}{ }_{2}^{\mathrm{III}} \mathrm{P}_{2} \mathrm{~W}_{16} \mathrm{O}_{60}\right)_{3}\right]^{26-}$ & $\left\{\mathrm{Co}_{6}\right\}$ & $\left\{\mathrm{P}_{2} \mathrm{~W}_{18}\right\}+$ Tris, pH 7, 常温 & {$[40]$} \\
\hline$\left[\mathrm{K}_{2}\left(\mathrm{Ni}_{2} \mathrm{P}_{2} \mathrm{~W}_{16} \mathrm{O}_{60}\right)_{3}\right]^{25-}$ & $\left\{\mathbf{N i}_{6}\right\}$ & $\left\{\mathrm{P}_{2} \mathrm{~W}_{18}\right\}+$ Tris, pH 7, 常温 & {$[41]$} \\
\hline$\left[\mathrm{Na}_{3}\left\{\mathrm{Co}\left(\mathrm{H}_{2} \mathrm{O}\right)_{4}\right\}_{6}\left\{\mathrm{WO}\left(\mathrm{H}_{2} \mathrm{O}\right)\right\}_{3}\left(\mathrm{P}_{2} \mathrm{~W}_{12} \mathrm{O}_{48}\right)_{3}\right]^{15-}$ & $\left\{\mathrm{Co}_{6} \mathbf{W}_{3}\right\}$ & $\mathrm{pH} 1$, 常温 & {$[67]$} \\
\hline$\left[\mathrm{Na}_{3}\left\{\mathrm{Ni}\left(\mathrm{H}_{2} \mathrm{O}\right)_{4}\right\}_{6}\left\{\mathrm{WO}\left(\mathrm{H}_{2} \mathrm{O}\right)\right\}_{3}\left(\mathrm{P}_{2} \mathrm{~W}_{12} \mathrm{O}_{48}\right)_{3}\right]^{15-}$ & $\left\{\mathbf{N i}_{6} \mathbf{W}_{3}\right\}$ & $\mathrm{pH} 1$, 常温 & {$[67]$} \\
\hline$\left[\mathrm{K}_{3}\left\{\mathrm{Mn}\left(\mathrm{H}_{2} \mathrm{O}\right)_{4}\right\}_{2}\left\{\mathrm{WO}_{2}\left(\mathrm{H}_{2} \mathrm{O}\right)_{2}\right\}_{2}\left\{\mathrm{WO}\left(\mathrm{H}_{2} \mathrm{O}\right)\right\}_{3}\left(\mathrm{P}_{2} \mathrm{~W}_{12} \mathrm{O}_{48}\right)_{3}\right]^{19-}$ & $\left\{\mathbf{M n}_{2} \mathbf{W}_{5}\right\}$ & $\mathrm{pH} 4$, 常温 & {$[35]$} \\
\hline$\left[\mathrm{Na}_{3}\left\{\mathrm{Ni}_{3.5}\left(\mathrm{H}_{2} \mathrm{O}\right)_{13}\right\}\left\{\mathrm{WO}_{2}\left(\mathrm{H}_{2} \mathrm{O}\right)_{2}\right\}_{2}\left\{\mathrm{WO}\left(\mathrm{H}_{2} \mathrm{O}\right)\right\}_{3}\left(\mathrm{P}_{2} \mathrm{~W}_{12} \mathrm{O}_{48}\right)_{3}\right]^{16-}$ & $\left\{\mathbf{N i}_{3.5} \mathbf{W}_{5}\right\}$ & $\mathrm{pH} 4$, 常温 & {$[35]$} \\
\hline$\left[\mathrm{Na}_{3}\left\{\mathrm{Cu}_{3}\left(\mathrm{H}_{2} \mathrm{O}\right)_{9}\right\}\left\{\mathrm{WO}_{2}\left(\mathrm{H}_{2} \mathrm{O}\right)_{2}\right\}_{2}\left\{\mathrm{WO}\left(\mathrm{H}_{2} \mathrm{O}\right)\right\}_{3}\left(\mathrm{P}_{2} \mathrm{~W}_{12} \mathrm{O}_{48}\right)_{3}\right]^{17-}$ & $\left\{\mathbf{C u}_{3} \mathbf{W}_{5}\right\}$ & $\mathrm{pH} 4$, 常温 & {$[35]$} \\
\hline$\left[\mathrm{K}_{3}\left\{\operatorname{GdMn}\left(\mathrm{H}_{2} \mathrm{O}\right)_{10}\right\}\left\{\mathrm{HMnGd}_{2}(\text { Tart }) \mathrm{O}_{2}\left(\mathrm{H}_{2} \mathrm{O}\right)_{15}\right\}\left\{\mathrm{P}_{6} \mathrm{~W}_{42} \mathrm{O}_{151}\left(\mathrm{H}_{2} \mathrm{O}\right)_{7}\right\}\right]^{11-}$ & $\left\{\mathbf{G d}_{3} \mathbf{M n}_{2}\right\}$ & $\mathrm{C}_{4} \mathrm{H}_{4} \mathrm{O}_{6} \mathrm{Na}_{2}, 40^{\circ} \mathrm{C}(3 \mathrm{~h})$ & {$[68]$} \\
\hline$\left[\mathrm{K}_{3}\left\{\mathrm{GdCo}\left(\mathrm{H}_{2} \mathrm{O}\right)_{11}\right\}_{2}\left\{\mathrm{P}_{6} \mathrm{~W}_{41} \mathrm{O}_{148}\left(\mathrm{H}_{2} \mathrm{O}\right)_{7}\right\}\right]^{13-}$ & $\left\{\mathrm{Gd}_{2} \mathrm{Co}_{2}\right\}$ & {$\left[\left(\mathrm{CH}_{3}\right)_{2} \mathrm{NH}_{2}\right] \mathrm{Cl}$, 常温 } & {$[68]$} \\
\hline$\left[\mathrm{Rb}_{33}\left\{\mathrm{~V}^{\mathrm{V}} \mathrm{V}_{3}^{\mathrm{IV}} \mathrm{O}_{7}\left(\mathrm{H}_{2} \mathrm{O}\right)_{6}\right\}_{2}\left\{\mathrm{H}_{6} \mathrm{P}_{6} \mathrm{~W}_{39} \mathrm{O}_{147}\left(\mathrm{H}_{2} \mathrm{O}\right)_{3}\right\}\right]^{15-}$ & $\left\{\mathbf{V}_{\mathbf{8}} \mathbf{W}_{3}\right\}$ & $\mathrm{NaVO}_{3}+\mathrm{VOSO}_{4}, \mathrm{pH} 3.4$, 常温 & {$[42]$} \\
\hline$\left[\left(\mathrm{Cr}\left(\mathrm{H}_{2} \mathrm{O}\right)_{2}\right)_{3}\left(\mathrm{H}_{2} \mathrm{P}_{2} \mathrm{~W}_{12} \mathrm{O}_{48}\right)_{3}\right]^{27-}$ & $\left\{\mathbf{C r}_{3}\right\}$ & pH 5.5, 常温 & {$[43]$} \\
\hline$\left[\mathrm{H}_{56} \mathrm{P}_{8} \mathrm{~W}_{48} \mathrm{Fe}_{28} \mathrm{O}_{248}\right]^{28-}$ & $\left\{\mathbf{F e}_{28}\right\}$ & $\mathrm{Fe}\left(\mathrm{ClO}_{3}\right)_{3}, \mathrm{pH} \mathrm{6}$, 回流1 h & {$[32]$} \\
\hline$\left[\left\{\mathrm{Sn}\left(\mathrm{CH}_{3}\right)_{2}\right\}_{4}\left(\mathrm{H}_{2} \mathrm{P}_{4} \mathrm{~W}_{24} \mathrm{O}_{92}\right)_{2}\right]^{28-}$ & $\left\{\mathbf{S n}_{4}\right\}$ & $\left(\mathrm{CH}_{3}\right)_{2} \mathrm{SnCl}_{2}, \mathrm{pH} 4.1,50^{\circ} \mathrm{C}(1 \mathrm{~h})$ & {$[44]$} \\
\hline$\left[\left\{\gamma-\mathrm{P}_{2} \mathrm{~W}_{12} \mathrm{O}_{48} \mathrm{Mn}_{4}\left(\mathrm{C}_{5} \mathrm{H}_{7} \mathrm{O}_{2}\right)_{2}\left(\mathrm{CH}_{3} \mathrm{CO}_{2}\right)\right\}_{6}\right]^{42-}$ & $\left\{\mathbf{M n}_{24}\right\}$ & $\left\{\mathrm{TBA}_{x} \mathrm{P}_{2} \mathrm{~W}_{12}\right\}+\mathrm{Mn}(\mathrm{acac})_{3}$, 于 $\mathrm{CH}_{2} \mathrm{Cl}_{2}$ 中结晶 & {$[45]$} \\
\hline$\left[\left\{\gamma-\mathrm{P}_{2} \mathrm{~W}_{12} \mathrm{O}_{48} \mathrm{Mn}_{4}\left(\mathrm{H}_{2} \mathrm{O}\right)_{6}\right\}_{4}\left(\mathrm{H}_{2} \mathrm{O}\right)_{4}\right]^{24-}$ & $\left\{\mathbf{M n}_{16}\right\}$ & $\left\{\mathbf{M n}_{24}\right\}+\mathrm{TBAOH}$, 于 $\mathrm{CH}_{2} \mathrm{Cl}_{2}$ 中结晶 & {$[45]$} \\
\hline
\end{tabular}

a) 如特殊条件中未提及, 默认合成原料为 $\left\{\mathrm{P}_{2} \mathrm{~W}_{12}\right\}$ 和金属无机盐; b) $\mathrm{M}=\mathrm{Mn} 、 \mathrm{Co} 、 \mathrm{Ni} 、 \mathrm{Cu}$ 或 $\left.\mathrm{Zn} ; \mathrm{c}\right) \mathrm{M}=\mathrm{Co} 、 \mathrm{Mn}$ 或Ni

$\left\{\mathbf{N b}_{32}\right\}$ (图2(b, c), 表1), 其中化合物 $\left\{\mathbf{N b}_{12}\right\}$ 和 $\left\{\mathbf{N b}_{32}\right\}$ 在相 同条件下的光催化析氢量分别可达 771 和 $1336 \mu \mathrm{mol} \mathrm{h}^{-1} \mathrm{~g}^{-1}$, 验证了 $\left\{\mathrm{P}_{2} \mathrm{~W}_{12}\right\}$ 多聚体的光催化析 氢活性随过渡金属核数与聚合数的增多而提升. 同时
根据四例阴离子簇的合成规律，作者还总结出以下结 论: (1) $\left[\mathrm{Nb}_{6} \mathrm{O}_{19}\right]^{8-}$ 离子在过氧化氢水溶液中分解, 因 此释放了 $\left\{\mathrm{NbO}_{2}\right\}$ 作为中间体进行反应; (2) 在 $\left\{\mathbf{M n}_{15}\right\}$ 六聚体中, $\mathrm{Mn}^{\mathrm{II}}$ 被氧化为 $\mathrm{Mn}^{\mathrm{III}}$, 并最终形成 $\left\{\mathrm{Mn}_{3}{ }_{3}^{\mathrm{II}}\right\}$ 簇; 
表 2 不同金属修饰的 $\left\{\mathbf{P}_{\mathbf{8}} \mathbf{W}_{48}\right\}$ 基多酸化合物

Table 2 Different metal-modified $\left\{\mathrm{P}_{8} \mathrm{~W}_{48}\right\}$-based POMs

\begin{tabular}{|c|c|c|c|}
\hline 阴离子簇的分子式 & 简称 & 特殊合成条件(原料/pH/加热) $\left.{ }^{\mathrm{a}}\right)$ & 文献 \\
\hline$\left[\mathrm{K}\left\{\mathrm{P}_{8} \mathrm{~W}_{48} \mathrm{O}_{184}\right\}\left\{\mathrm{V}_{4}{ }_{4} \mathrm{~V}^{\mathrm{IV}}{ }_{2} \mathrm{O}_{12}\left(\mathrm{H}_{2} \mathrm{O}\right)_{2}\right\}\right]^{24-}$ & $\left\{V_{12}\right\}$ & $\mathrm{VOSO}_{4}, \mathrm{pH} 4.4,50^{\circ} \mathrm{C}(4 \mathrm{~h})$ & {$[50]$} \\
\hline$\left[\mathrm{K}_{8}\left(\mathrm{P}_{8} \mathrm{~W}_{48} \mathrm{O}_{184}\right)\left(\mathrm{Mo}^{\mathrm{VI}} \mathrm{O}_{2}\right)_{4}\left(\mathrm{Mo}_{4}^{\mathrm{V}} \mathrm{O}_{13}\right)_{2}\right]^{24-}$ & $\left\{\mathbf{M o}_{12}\right\}$ & $\mathrm{Na}_{2} \mathrm{~S}_{2} \mathrm{O}_{4}+\mathrm{N}_{2}$ 保护, $\mathrm{pH} 4.4,60^{\circ} \mathrm{C}(3 \mathrm{~h})$ & {$[51]$} \\
\hline$\left[\mathrm{K}_{4}\left\{\mathrm{Mo}_{4} \mathrm{O}_{4} \mathrm{~S}_{4}\left(\mathrm{H}_{2} \mathrm{O}\right)_{3}(\mathrm{OH})_{2}\right\}_{2}\left(\mathrm{WO}_{2}\right)\left(\mathrm{P}_{8} \mathrm{~W}_{48} \mathrm{O}_{184}\right)\right]^{30-}$ & $\left\{\mathbf{M o}_{8} \mathrm{~S}_{8}\right\}$ & $\begin{array}{c}\mathrm{K}_{2-x}\left(\mathrm{NMe}_{4}\right)_{x}\left[\mathrm{I}_{2} \mathrm{Mo}_{10} \mathrm{O}_{10} \mathrm{~S}_{10}(\mathrm{OH})_{10}\left(\mathrm{H}_{2} \mathrm{O}\right)_{5}\right] \cdot 20 \mathrm{H}_{2} \mathrm{O}, \mathrm{pH} 2 \\
60^{\circ} \mathrm{C}(4 \mathrm{~h})\end{array}$ & {$[52]$} \\
\hline$\left[\left(\mathrm{MoO}_{2}\right)_{2}\left(\mathrm{P}_{8} \mathrm{~W}_{48} \mathrm{O}_{184}\right)\right]^{38-}$ & $\left\{\mathbf{M o}_{2}\right\}$ & {$\left[\mathrm{Mo}_{2} \mathrm{O}_{4}\left(\mathrm{H}_{2} \mathrm{O}\right)_{6}\right]^{2+}, \mathrm{pH} 4,95^{\circ} \mathrm{C}(2 \mathrm{~h})$} & {$[70]$} \\
\hline$\left[\left\{\mathrm{M}_{2}\left(\mathrm{OH}_{2}\right)_{2}\right\}_{2}\left\{\mathrm{M}\left(\mathrm{OH}_{2}\right)_{2}\right\}_{4} \mathrm{P}_{8} \mathrm{~W}_{48} \mathrm{O}_{176}\left(\mathrm{OCH}_{3}\right)_{8}\right]^{16-}$ & $\left\{\mathbf{M}_{8}\right\}^{\text {b) }}$ & 乙腈/二氯甲烷溶剂 $+\mathrm{TBAOH}+\left\{\mathrm{TBA}_{12} \mathrm{P}_{8} \mathrm{~W}_{48}\right\}$, 常温 & {$[63]$} \\
\hline$\left[\mathrm{Mn}_{8}\left(\mathrm{H}_{2} \mathrm{O}\right)_{48} \mathrm{P}_{8} \mathrm{~W}_{48} \mathrm{O}_{184}\right]^{24-}$ & $\left\{\mathbf{M n}_{8}\right\}$ & $\mathrm{Mn}\left(\mathrm{ClO}_{4}\right)_{2}, \mathrm{pH} 4,90^{\circ} \mathrm{C}(20 \mathrm{~h})$, 冷却至常温后 $4^{\circ} \mathrm{C}$ 结晶 & {$[53]$} \\
\hline$\left[\mathrm{Mn}_{14}\left(\mathrm{H}_{2} \mathrm{O}\right)_{30} \mathrm{P}_{8} \mathrm{~W}_{48} \mathrm{O}_{184}\right]^{12-}$ & $\left\{\mathbf{M n}_{14}\right\}$ & $\mathrm{Mn}\left(\mathrm{ClO}_{4}\right)_{2}, \mathrm{pH} 4$, 常温 & {$[54]$} \\
\hline$\left[\mathrm{K}_{10} \mathrm{Li}_{4} \mathrm{NaMn}_{10}\left(\mathrm{~W}_{4} \mathrm{O}_{16}\right)\left(\mathrm{H}_{2} \mathrm{O}\right)_{50}\left(\mathrm{P}_{8} \mathrm{~W}_{48} \mathrm{O}_{184}\right) \mathrm{Cl}_{2}\right]^{15-}$ & $\left\{\mathbf{M n}_{10} \mathbf{W}_{4}\right\}$ & $\mathrm{Mn}\left(\mathrm{ClO}_{4}\right)_{2}, \mathrm{pH} 8,85^{\circ} \mathrm{C}(1 \mathrm{~h})$ & {$[71]$} \\
\hline$\left[\left(\mathrm{P}_{8} \mathrm{~W}_{48} \mathrm{O}_{184}\right)\left\{\left(\mathrm{P}_{2} \mathrm{~W}_{14} \mathrm{Mn}_{4} \mathrm{O}_{60}\right)\left(\mathrm{P}_{2} \mathrm{~W}_{15} \mathrm{Mn}_{3} \mathrm{O}_{58}\right)_{2}\right\}_{4}\right]^{144-}$ & $\left\{\mathbf{M n}_{40}\right\}$ & $\left\{\mathrm{P}_{2} \mathrm{~W}_{12}\right\},\left[\mathrm{Mn}_{12} \mathrm{O}_{12}\left(\mathrm{O}_{2} \mathrm{CMe}\right)_{16}\left(\mathrm{H}_{2} \mathrm{O}\right)_{40}\right], \mathrm{pH} 4.4$, 常温 & {$[55]$} \\
\hline$\left[\mathrm{P}_{8} \mathrm{~W}_{48} \mathrm{O}_{184} \mathrm{Fe}_{16}(\mathrm{OH})_{28}\left(\mathrm{H}_{2} \mathrm{O}\right)_{4}\right]^{20-}$ & $\left\{\mathbf{F e}_{16}\right\}$ & $\mathrm{H}_{2} \mathrm{O}_{2}, \mathrm{pH} 4,80^{\circ} \mathrm{C}(1 \mathrm{~h})$ & {$[56]$} \\
\hline$\left[\mathrm{Co}_{10}\left(\mathrm{H}_{2} \mathrm{O}\right)_{34}\left(\mathrm{P}_{8} \mathrm{~W}_{48} \mathrm{O}_{184}\right)\right]^{20-}$ & $\left\{\mathrm{Co}_{10} \mathbf{a}\right\}$ & $\mathrm{pH} 5.75,70^{\circ} \mathrm{C}(20 \mathrm{~h})$, 冷却至常温后 $4^{\circ} \mathrm{C}$ 结晶 & {$[49]$} \\
\hline$\left[\mathrm{Co}_{10}\left(\mathrm{H}_{2} \mathrm{O}\right)_{44}\left(\mathrm{P}_{8} \mathrm{~W}_{48} \mathrm{O}_{184}\right)\right]^{20-}$ & $\left\{\mathbf{C o}_{10} \mathbf{b}\right\}$ & $\mathrm{pH} 5.75,70^{\circ} \mathrm{C}(20 \mathrm{~h})$, 冷却至常温 & {$[49]$} \\
\hline $\mathrm{Co}_{5}\left[\mathrm{Co}_{5.5}\left(\mathrm{H}_{2} \mathrm{O}\right)_{19} \mathrm{P}_{8} \mathrm{~W}_{48.5} \mathrm{O}_{184}\right]^{16-}$ & $\left\{\mathrm{Co}_{10.5}\right\}$ & $\mathrm{NaClO}_{4}, \mathrm{pH} 3.7$, 常温 & {$[72]$} \\
\hline $\mathrm{Co}_{5}\left[\mathrm{Co}_{7}\left(\mathrm{H}_{2} \mathrm{O}\right)_{28} \mathrm{P}_{8} \mathrm{~W}_{48} \mathrm{O}_{184}\right]^{16-}$ & $\left\{\mathrm{Co}_{12}\right\}$ & $\mathrm{pH} 3.4$, 常温 & {$[72]$} \\
\hline $\mathrm{Co}_{11}\left[\mathrm{Co}_{8}\left(\mathrm{H}_{2} \mathrm{O}\right)_{32} \mathrm{P}_{8} \mathrm{~W}_{48} \mathrm{O}_{184}\right]^{2-}$ & $\left\{\mathbf{C o}_{19}\right\}$ & $\mathrm{pH} 2.7$, 常温 & {$[72]$} \\
\hline$\left[\left\{\mathrm{K}\left(\mathrm{H}_{2} \mathrm{O}\right)\right\}_{3}\left\{\mathrm{Ru}(p \text {-cymene })\left(\mathrm{H}_{2} \mathrm{O}\right)\right\}_{4} \mathrm{P}_{8} \mathrm{~W}_{49} \mathrm{O}_{186}\left(\mathrm{H}_{2} \mathrm{O}\right)_{2}\right]^{27-}$ & $\left\{\mathbf{R u}_{4}\right\}$ & {$\left[\mathrm{Ru}(p \text {-cymene }) \mathrm{Cl}_{2}\right]_{2}, \mathrm{pH} 6,80^{\circ} \mathrm{C}(1 \mathrm{~h})$} & {$[73]$} \\
\hline$\left[\alpha \gamma \alpha \gamma-\mathrm{P}_{8} \mathrm{~W}_{48} \mathrm{O}_{184}\left\{\mathrm{Cu}\left(\mathrm{H}_{2} \mathrm{O}\right)\right\}_{2}\right]^{36-}$ & $\left\{\mathbf{C u}_{2} \mathbf{a}\right\}$ & $\mathrm{LiCl}, \mathrm{pH} 5.2,70^{\circ} \mathrm{C}(3 \mathrm{~h})$ & {$[74]$} \\
\hline$\left[\gamma \gamma \gamma \gamma-\mathrm{P}_{8} \mathrm{~W}_{48} \mathrm{O}_{184}\left\{\mathrm{Cu}\left(\mathrm{H}_{2} \mathrm{O}\right)_{0.5}\right\}_{4}\right]^{32-}$ & $\left\{\mathbf{C u}_{2} \mathbf{b}\right\}$ & $\mathrm{CuCl}_{2}$ 翻倍, $\mathrm{pH} 5.2,70^{\circ} \mathrm{C}(3 \mathrm{~d})$ & {$[74]$} \\
\hline$\left[\alpha \gamma \gamma \gamma-\mathrm{P}_{8} \mathrm{~W}_{48} \mathrm{O}_{184}\left\{\mathrm{Cu}\left(\mathrm{H}_{2} \mathrm{O}\right)\right\}_{3}\right]^{34-}$ & $\left\{\mathrm{Cu}_{2} \mathbf{c}\right\}$ & $\mathrm{CuCl}_{2}$ 与 $\left\{\mathrm{P}_{8} \mathrm{~W}_{48}\right\}$ 翻倍, $\mathrm{pH} 5.2,70^{\circ} \mathrm{C}(8 \mathrm{~d})$ & {$[74]$} \\
\hline$\left[\mathrm{Cu}_{2}\left(\mathrm{H}_{2} \mathrm{O}\right)_{2}\left\{\mathrm{Cu}_{4}(\mathrm{OH})_{4}\left(\mathrm{H}_{2} \mathrm{O}\right)_{8}\right\}_{2}\left(\mathrm{P}_{8} \mathrm{~W}_{48} \mathrm{O}_{184}\right)\right]^{28-}$ & $\left\{\mathbf{C u}_{10}\right\}$ & {$\left[\mathrm{Mo}_{2} \mathrm{O}_{4}\left(\mathrm{H}_{2} \mathrm{O}\right)_{6}\right]^{2+}, \mathrm{pH} 4,95^{\circ} \mathrm{C}(2 \mathrm{~h})$} & {$[70]$} \\
\hline$\left[\mathrm{Cu}_{20} \mathrm{Cl}(\mathrm{OH})_{24}\left(\mathrm{H}_{2} \mathrm{O}\right)_{12}\left(\mathrm{P}_{8} \mathrm{~W}_{48} \mathrm{O}_{184}\right)\right]^{25-}$ & $\left\{\mathbf{C u}_{20}\right\}$ & $\mathrm{pH} 6,80^{\circ} \mathrm{C}(1 \mathrm{~h})$ & {$[47]$} \\
\hline$\left[\mathrm{Cu}_{20}\left(\mathrm{~N}_{3}\right)_{6}(\mathrm{OH})_{18} \mathrm{P}_{8} \mathrm{~W}_{48} \mathrm{O}_{184}\right]^{24-}$ & $\left\{\mathrm{Cu}_{20}\right.$-azido $\}$ & $\mathrm{NaN}_{3}, 80^{\circ} \mathrm{C}(15 \mathrm{~min})$, 冷却至常温后 $3^{\circ} \mathrm{C}$ 结晶 & {$[48]$} \\
\hline$\left[\mathrm{Al}_{16}(\mathrm{OH})_{24}\left(\mathrm{H}_{2} \mathrm{O}\right)_{8}\left(\mathrm{P}_{8} \mathrm{~W}_{48} \mathrm{O}_{184}\right)\right]^{16-}$ & $\left\{\mathbf{A l}_{16}\right\}$ & $\mathrm{pH} 4,80^{\circ} \mathrm{C}(60 \mathrm{~h})$ & {$[57]$} \\
\hline$\left[\left(\mathrm{PhAs}^{\mathrm{V}} \mathrm{O}\right)_{4} \mathrm{P}_{8} \mathrm{~W}_{48} \mathrm{O}_{184}\right]^{32-}$ & $\left\{(\mathrm{PhAs})_{4}\right\}$ & $\mathrm{PhAsO}_{3} \mathrm{H}_{2}+\left[\left(\mathrm{CH}_{3}\right)_{2} \mathrm{NH}_{2}\right] \mathrm{Cl}, \mathrm{pH} 3,60^{\circ} \mathrm{C}(4 \mathrm{~d})$ & {$[58]$} \\
\hline$\left[\left(p-\mathrm{NH}_{2} \mathrm{C}_{6} \mathrm{H}_{4} \mathrm{As}^{\mathrm{v}} \mathrm{O}\right)_{4} \mathrm{P}_{8} \mathrm{~W}_{48} \mathrm{O}_{184}\right]^{32-}$ & $\left\{\left(\mathrm{NH}_{2} \mathrm{PhAs}\right)_{4}\right\}$ & $p-\left(\mathrm{H}_{2} \mathrm{~N}\right) \mathrm{PhAsO} \mathrm{A}_{3} \mathrm{H}_{2}+\left[\left(\mathrm{CH}_{3}\right)_{2} \mathrm{NH}_{2}\right] \mathrm{Cl}, \mathrm{pH} 3,60^{\circ} \mathrm{C}(4 \mathrm{~d})$ & {$[58]$} \\
\hline$\left[\left(\mathrm{H}_{3} \mathrm{NC}_{6} \mathrm{H}_{4} \mathrm{AsO}\right)_{3} \mathrm{P}_{8} \mathrm{~W}_{48} \mathrm{O}_{184} \mathrm{H}_{x}\left\{\mathrm{WO}_{2}\left(\mathrm{H}_{2} \mathrm{O}\right)_{2}\right\}_{0.4}\right]^{(30.2-x)-}$ & $\left\{\left(\mathrm{NH}_{2} \mathrm{PhAs}\right)_{4} \mathrm{a}\right\}$ & $\left\{\left(\mathbf{N H}_{2} \mathbf{P h A s}\right)_{4}\right\}, \mathrm{pH} 2.7,70^{\circ} \mathrm{C}(40 \mathrm{~min})$ & {$[58]$} \\
\hline$\left[(\mathrm{SeO})_{4} \mathrm{P}_{8} \mathrm{~W}_{48} \mathrm{O}_{184}\right]^{32-}$ & $\left\{\mathrm{Se}_{4}\right\}$ & $\mathrm{SeO}_{2}, \mathrm{pH} 4.8,80^{\circ} \mathrm{C}(1 \mathrm{~h})$ & {$[75]$} \\
\hline$\left[\mathrm{Ln}_{4}\left\{\mathrm{KP}_{8} \mathrm{~W}_{48} \mathrm{O}_{184}\left(\mathrm{H}_{4} \mathrm{~W}_{4} \mathrm{O}_{12}\right)_{2} \mathrm{Ln}_{2}\left(\mathrm{H}_{2} \mathrm{O}\right)_{10}\right\}\right]^{13-}$ & $\left\{\mathbf{L n}_{6}\right\}^{\mathrm{c}}$ & $\mathrm{pH} 1.5,140^{\circ} \mathrm{C}$ 水热过夜 & {$[76]$} \\
\hline
\end{tabular}

a) 如特殊条件中未提及, 默认合成原料为 $\left\{\mathrm{P}_{8} \mathrm{~W}_{48}\right\}$ 及简单金属无机盐; b) M=Mn、Co、Ni、Cu或 $\left.\mathrm{Zn} ; \mathrm{c}\right) \mathrm{Ln}=\mathrm{La} 、 \mathrm{Ce} 、 \mathrm{Pr}$ 或 $\mathrm{Nd}$

(3) 在酸性溶液中, O-O键会随着加热逐步裂解并缩合 为 Nb-O-Mn桥, 从而稳定结构中的高电荷组分.

Mo、 $\mathrm{W}$ 和 $\mathrm{V}$ 是 $\mathrm{POMs}$ 的主要构成金属，其酸强度 按 $\mathrm{W}>\mathrm{Mo}>\mathrm{V}$ 顺序排列, 相应的氧化能力则按 $\mathrm{V}>\mathrm{Mo}>\mathrm{W}$ 顺序排列．不同于使用 $\left\{\mathrm{Nb}_{6}\right\}$ 修饰填补 $\left\{\mathrm{P}_{2} \mathrm{~W}_{12}\right\}$ 中的缺 位，使用 $\mathrm{V}$ 对缺位进行填补时使用 $\mathrm{NaVO}_{3}$ 为原料，相关 研究发表于Hervé研究组 ${ }^{[62]}$ 在2004年的报道中. 在该项 研究中, 化合物 $\left\{\mathrm{P}_{2} \mathrm{~W}_{12} \mathrm{~V}_{6}\right\}$ 在 $\mathrm{K}_{12}\left[\mathrm{H}_{2} \mathrm{P}_{2} \mathrm{~W}_{12} \mathrm{O}_{48}\right] \cdot 24 \mathrm{H}_{2} \mathrm{O}$ 与
$\mathrm{NaVO}_{3}$ 的盐酸混合溶液中蒸发结晶获得，然而尽管文 献提供了化合物的合成步骤，但并没有得到具体的 晶体结构数据. 同时, 他们使用 ${ }^{31} \mathrm{P}$ 和 ${ }^{183} \mathrm{~W}$ 核磁对 $\left\{\mathbf{P}_{2} \mathbf{W}_{12} \mathbf{V}_{6}\right\}$ 进行了结构预测, 并讨论了其在溶液中的稳 定性. 结果显示, 此化合物在 $1 \mathrm{M}$ 的 $\mathrm{LiCl}$ 溶液中并不稳 定，其 ${ }^{31} \mathrm{P}$ 核磁结果会逐渐由单一信号分裂为两个等强 度信号(这可能是赤道位 $\mathrm{V}$ 原子通过迁移到其他赤道 位，以分散骨架的负电荷所致), 通常此过程在室温下 
需要一周时间完成, 煮沸条件下则需要约 $15 \mathrm{~h}$, 缓慢的 迁移过程导致无法形成符合单晶测试标准的样品. 此 外, 其他过渡金属离子也可引入到合成体系中, 以实现 对 $\left\{\mathrm{P}_{2} \mathrm{~W}_{12}\right\}$ 缺位的修饰. 例如, Gouzerh研究组 ${ }^{[32]}$ 于 2005 年报道的化合物 $\left\{\mathrm{Fe}_{9}\right\}\left(\mathrm{K}_{4} \mathrm{Li}_{2}\left[\mathrm{H}_{4} \mathrm{P}_{2} \mathrm{~W}_{12} \mathrm{Fe}_{9} \mathrm{O}_{56}(\mathrm{OAc})_{7}\right]\right.$, 图 3 (a), 表1). 在该阴离子簇中, $\mathrm{Fe}$ 吕首先对 $\left\{\mathrm{P}_{2} \mathrm{~W}_{12}\right\}$ 模块进 行补位, 使形成饱和的 $\left\{\mathrm{P}_{2} \mathrm{Fe}_{6} \mathrm{~W}_{12}\right\}$ 阴离子簇, 剩余的 3 个 Fe $\mathrm{e}^{\mathrm{III}}$ 则通过 $\mathrm{OAc}$ 的桥联，以盖帽形式固定在簇表面; 后续循环伏安测试结果显示, $\mathrm{Fe}$ 元素的修饰会使 $\left\{\mathrm{P}_{2} \mathrm{~W}_{12}\right\}$ 表现出较宽的还原峰, 这个可以归因于组分中 $\mathrm{Fe}^{\mathrm{III}}$ 还原为 $\mathrm{Fe}^{\mathrm{II}}$ 的现象, 在夹心化合物 $\alpha \beta \beta \alpha-\left[\left(\mathrm{Fe}^{\mathrm{III}} \mathrm{OH}_{2}\right)_{2}-\right.$ $\left.\mathrm{Fe}^{\mathrm{III}}{ }_{2}\left(\mathrm{P}_{2} \mathrm{~W}_{15} \mathrm{O}_{56}\right)_{2}\right]_{12}$ 中是无法观测到的; 另外, 由于 9 个 $\mathrm{Fe}^{\mathrm{III}}$ 中心间较强的反铁磁耦合, 化合物所表现出的磁 现象比预期结果要差.

作为一种高度缺位的阴离子簇, $\left\{\mathrm{P}_{2} \mathrm{~W}_{12}\right\}$ 构筑模块 在水溶液处于亚稳态, 合成过程时常伴随着结构解离 和重组的发生, 为防止上述状况的出现, 并使合成产 物更加洁净单一, 使用有机溶剂取代水溶液是另外一 种合成策略 ${ }^{[3,45,63]}$. 在Suzuki研究组 ${ }^{[3,45,63]}$ 的研究中, 乙腈被证明是一种优良的多酸合成溶剂: 通过将 $\mathrm{M}(\mathrm{OAc})_{2}$ 与 $\left\{\mathrm{P}_{2} \mathrm{~W}_{12}\right\}$ 的 TBA盐混合于热乙腈溶液中, 经 过长时间搅拌和沉淀重结晶后, 可以得到由 2 个 $\mathrm{M}^{2+}$ 、

1 个 OAc 和 2 个 $\mathrm{CH}_{3} \mathrm{CONH}$ 基团修饰的 $\left\{\mathrm{P}_{2} \mathrm{~W}_{12}\right\}$ 基化合物 $\mathrm{TBA}_{5}\left[\gamma-\mathrm{P}_{2} \mathrm{~W}_{12} \mathrm{O}_{44} \mathrm{M}_{2}(\mathrm{OAc})\left(\mathrm{CH}_{3} \mathrm{CONH}\right)_{2}\right] \cdot 7 \mathrm{H}_{2} \mathrm{O} \cdot \mathrm{CH}_{3} \mathrm{CN}$ $\left(\left\{\mathbf{M}_{2}\right\}, \mathrm{M}=\mathrm{Mn}^{2+} 、 \mathrm{Co}^{2+} 、 \mathrm{Ni}^{2+} 、 \mathrm{Cu}^{2+}\right.$ 或 $\mathrm{Zn}^{2+}$, 图3(b), 表 $1)^{[34]}$. $\left\{\mathbf{M}_{2}\right\}$ 的阴离子簇与 $\left\{\mathrm{Fe}_{9}\right\}$ 有所不同, 该结构中的 $\mathrm{M}^{2+}$ 以五配位的类金字塔构型存在: 每个 $\mathrm{M}^{2+}$ 分别与两 个 $\left\{\mathrm{PO}_{4}\right\}$ 单元中的氧原子、 $\mathrm{POM}$ 骨架缺位的两个氧原 子和OAc配体的一个氧原子配位; 在此基础上, 两个 “类金字塔”间以共边形式并连, 通过OAc桥联配体稳 定骨架. 处于边缘位置的两个缺位则被 $\mathrm{CH}_{3} \mathrm{CONH}$ 配 体封端，以抑制高反应性空位处的不希望的低聚反应, 该配体可能是乙腈的水合产物. 另外, 二价过渡金属阳 离子 $\left(\mathrm{M}(\mathrm{OAc})_{2}\right)$ 也可用于合成此类结构, 但当使用三价 阳离子 $\left(\right.$ 如 $\mathrm{Mn}(\mathrm{OAc})_{3} 、 \mathrm{Fe}(\mathrm{acac})_{3}$ 或 $\left.\mathrm{Co}(\mathrm{OAc})_{3}\right)$ 时, 则无 法得到双核结构, 这表明过渡金属离子在 $\left\{\mathrm{P}_{2} \mathrm{~W}_{12}\right\}$ 缺位 上的配位填充是有一定条件限制的.

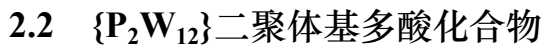

对于 $\left\{\mathrm{P}_{2} \mathrm{~W}_{12}\right\}$ 构筑模块而言, 由于其六缺位集中 于同侧, 使模块具有较高的负电荷数和较多的含氧缺
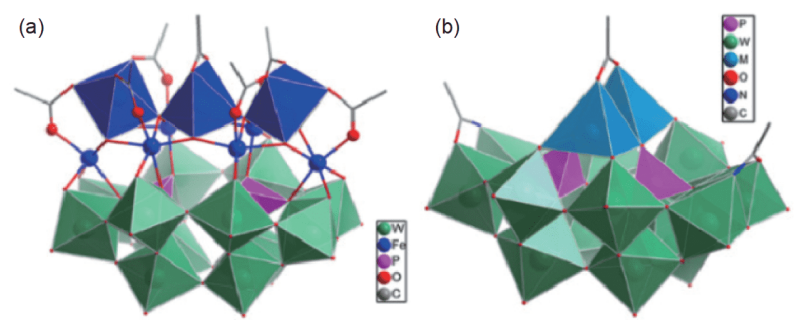

图 3 (a) $\left\{\mathrm{Fe}_{9}\right\}$ 和(b) $\left\{\mathrm{M}_{2}\right\}$ 修饰的 $\left\{\mathrm{P}_{2} \mathrm{~W}_{12}\right\}$ 基多金属氧酸盐示 意图 ${ }^{[31]}$ (网络版彩图)

Figure 3 Structural illustration of (a) $\left\{\mathrm{Fe}_{9}\right\}$ and (b) $\left\{\mathbf{M}_{2}\right\}$-modified $\left\{\mathrm{P}_{2} \mathrm{~W}_{12}\right\}$ monomer-based POMs [31] (color online).

位，故而模块间很难通过自组装形式进行多聚 ( $\left\{\mathrm{P}_{4} \mathrm{~W}_{48}\right\}$ 除外). 已报道的多聚体，大都是模块间通过 过渡金属/主族金属离子桥联组装而成的, 金属离子间 通过过渡金属/主族金属离子桥联组装而成的, 金属离 子的修饰位置和数量将会显著影响所形成的多聚体 的构型. 例如, 在Gouzerh等 ${ }^{[32]}$ 的研究中, 通过将 $\mathrm{Fe}$ $\left(\mathrm{NO}_{3}\right)_{2}$ 与 $\left\{\mathrm{P}_{2} \mathrm{~W}_{12}\right\}$ 模块混合溶解于热 $\mathrm{KOH}$ 溶液, 可以 冷却结晶得到二聚体化合物: $\mathrm{Na}_{10} \mathrm{~K}_{6}\left[\mathrm{H}_{12} \mathrm{Fe}_{8} \mathrm{P}_{4} \mathrm{~W}_{28}\right.$

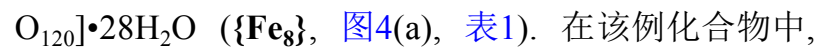
$\left\{\mathrm{P}_{2} \mathrm{~W}_{12}\right\}$ 的极缺位和赤道缺位分别被 $\left\{\mathrm{WO}_{6}\right\}$ 和 $\left\{\mathrm{FeO}_{6}\right\}$ 填充, 8 个 $\left\{\mathrm{FeO}_{6}\right\}$ 通过共用 $\mu_{2}$-氧相互连接, 形成 $\left\{\mathrm{Fe}_{8}\right\}$ 八元金属氧簇，用于补位的 $\mathrm{W}$ 可能由模块分解生成; 而当原料中的硝酸盐换成簇合物 $\left[\mathrm{Fe}^{\mathrm{III}}{ }_{2} \mathrm{M}{ }^{\mathrm{II}} \mathrm{O}(\mathrm{OAc})_{6}\right.$ $\left.\left(\mathrm{H}_{2} \mathrm{O}\right)_{3}\right](\mathrm{M}=\mathrm{Co} 、 \mathrm{Mn}$ 或 $\mathrm{Ni})$ 时, 产物将会变为 $\mathrm{K}_{12}\{\mathrm{M}$ $\left.\left(\mathrm{H}_{2} \mathrm{O}\right)_{4}\right\}_{2}\left\{\mathrm{H}_{12} \mathrm{Fe}_{8} \mathrm{P}_{4} \mathrm{~W}_{28} \mathrm{O}_{120}\right\} \cdot 30 \mathrm{H}_{2} \mathrm{O} \quad\left(\left\{\mathbf{F e}_{8} \mathbf{M}_{2}\right\}\right.$, 图4(b), 表1), 由于 $\mathrm{M}^{\mathrm{II}}$ 与 $\mathrm{Fe}^{\mathrm{III}}$ 的离子半径不同, 可以通过比较 其与 $\mathrm{O}$ 原子的成键键长以实现两者的区分. 具有类似 阴离子簇的多酸化合物 $\left\{\mathbf{M n}_{5} \mathbf{W}_{4}\right\}$ 而后被王恩波团 队 ${ }^{[64]}$ 报道, 化合物可在均苯四甲酸二酐(PMDA)作为 模板剂的条件下通过水热法合成, 其分子式为: $\left\{\mathrm{Na}_{8}\right.$ $\mathrm{H}_{2} \mathrm{PMDA}\left(\mathrm{H}_{2} \mathrm{enMe}\right)_{4}\left\{\mathrm{Mn}\left(\mathrm{H}_{2} \mathrm{O}\right)_{2}\left[\left(\mathrm{~W}_{4} \mathrm{Mn}_{4} \mathrm{O}_{12}\right)\left(\mathrm{P}_{2} \mathrm{~W}_{14}\right.\right.\right.$ $\left.\left.\left.\mathrm{O}_{54}\right)_{2}\right]\right\} \cdot 17 \mathrm{H}_{2} \mathrm{O}$ (图4(c), 表1). 在该例化合物中, $\left\{\mathrm{P}_{2} \mathrm{~W}_{12}\right\}$ 的四个赤道缺位被无序的 $\mathrm{W}$ 和 $\mathrm{Mn}$ 填充, 两者 各持 $1 / 2$ 占有率; 后 $\left\{\mathrm{P}_{2} \mathrm{~W}_{16} \mathrm{Mn}_{2}\right\}$ 以“肩并肩”形式组成 以 $\left\{\mathrm{Mn}_{4} \mathrm{~W}_{4}\right\}$ 为核的二聚体, 相邻二聚体间通过 $\left\{\mathrm{MnO}_{6}\right\}$ 单元连接, 形成沿 $c$ 方向伸展的无限一维链.

除上述通过八元过渡金属氧簇，以“肩并肩”形式 连接构成的二聚体外, $\left\{\mathrm{P}_{2} \mathrm{~W}_{12}\right\}$ 还能在 $\mathrm{K}^{+}$的诱导下形 成“心型”二聚体, 空余缺位的修饰使之形成“缺位Preyssler”型阴离子簇 $\left[\mathrm{P}_{4} \mathrm{~W}_{24} \mathrm{O}_{92}\right]^{20-}$. 例如, 在王恩波团队 ${ }^{[35]}$ 

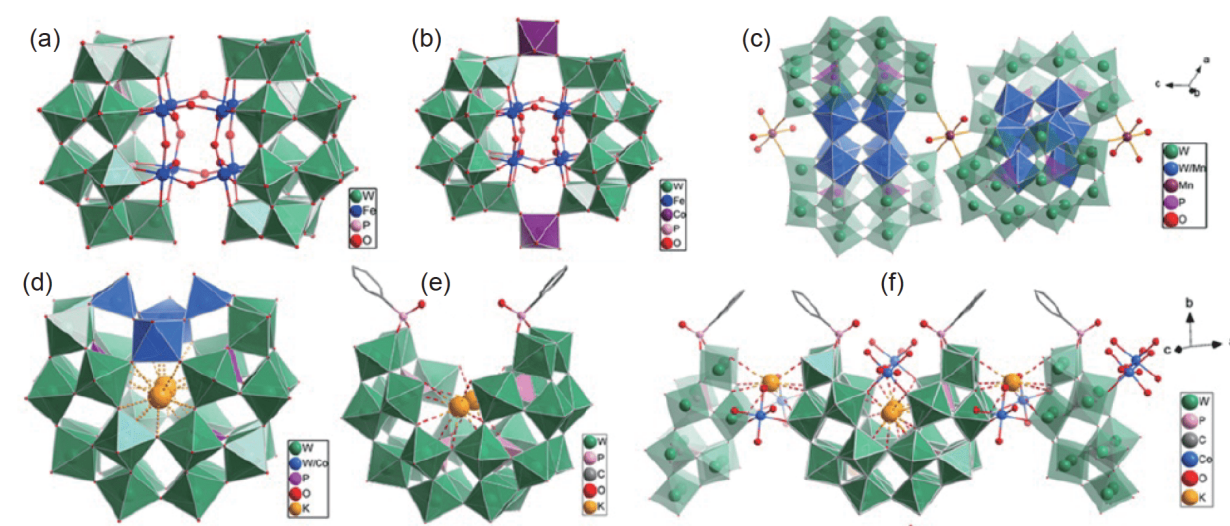

(e)
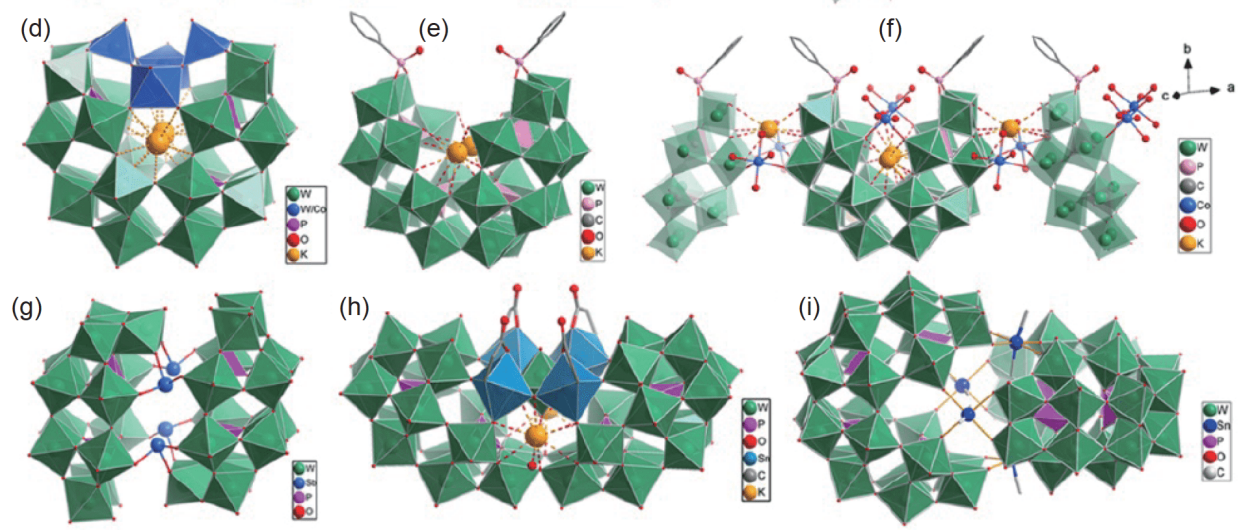

图 4 (a i) 不同金属(按顺序依次为 $\left\{\mathrm{Fe}_{8}\right\} 、\left\{\mathrm{Fe}_{8} \mathrm{M}_{2}\right\} 、\left\{\mathrm{Mn}_{5} \mathbf{W}_{4}\right\} 、\left\{\mathrm{Co}_{2} \mathbf{W}_{2}\right\} 、\left\{(\mathrm{PhPO})_{2}\right\} 、\left\{\mathrm{Co}_{3}\left(\mathrm{PhPO}_{2}\right\} 、\left\{\mathrm{Sb}_{4}\right\} 、\left\{\mathrm{Sn}_{4} \mathbf{W}\right\}\right.$ 和 $\left\{\mathbf{S n}_{4}\right\}$ )修饰的 $\left\{\mathrm{P}_{2} \mathrm{~W}_{12}\right\}$ 二聚体/四聚体基多酸化合物结构示意图(网络版彩图)

Figure 4 (a-i) Structural illustration of different metal $\left(\left\{\mathbf{F e}_{8}\right\},\left\{\mathbf{F e}_{8} \mathbf{M}_{2}\right\},\left\{\mathbf{M n}_{5} \mathbf{W}_{4}\right\},\left\{\mathbf{C o}_{2} \mathbf{W}_{2}\right\},\left\{(\mathbf{P h P O})_{2}\right\},\left\{\mathbf{C o}_{3}(\mathbf{P h P O})_{2}\right\},\left\{\mathbf{S b}_{4}\right\},\left\{\mathbf{S n}_{4} \mathbf{W}\right\}\right.$, and $\left\{\mathbf{S n}_{4}\right\}$, respectively) modified $\left\{\mathrm{P}_{2} \mathrm{~W}_{12}\right\}$ dimer- or tetramer-based POMs (color online).

的研究中, $\left\{\mathrm{P}_{2} \mathrm{~W}_{12}\right\}$ 构筑模块可以在煮沸的 $\mathrm{NaCl}$ 溶液中 与 $\mathrm{CoCl}_{2}$ 结合, 形成化合物 $\mathrm{K}_{3} \mathrm{Na}_{17}\left[\left\{\mathrm{~W}_{2} \mathrm{Co}_{2} \mathrm{O}_{8}\left(\mathrm{H}_{2} \mathrm{O}\right)_{2}\right\}-\right.$ $\left.\left(\mathrm{P}_{2} \mathrm{~W}_{12} \mathrm{O}_{46}\right)_{2}\right] \cdot 30 \mathrm{H}_{2} \mathrm{O}\left(\left\{\mathbf{C o}_{2} \mathbf{W}_{2}\right\}\right.$, 图4(d), 表1). 遗憾的 是，尽管 $\left\{\mathrm{P}_{4} \mathrm{~W}_{24}\right\}$ 构筑模块可以被合成并通过质谱表 征, 但是由于 $\left\{\mathrm{P}_{4} \mathrm{~W}_{24}\right\}$ 聚阴离子的稳定性较差, 故与 $\left\{\mathrm{P}_{2} \mathrm{~W}_{12}\right\}$ 类似, 直到今天仍未得到具有明确单晶数据的 晶体结构 ${ }^{[69]}$. 不过Kögerler课题组 ${ }^{[36]}$ 研究发现, $\left\{\mathrm{P}_{4} \mathrm{~W}_{24}\right\}$ 阴离子簇可通过有机膦修饰以稳定结构, 生成 化合物 $\mathrm{K}_{3.5} \mathrm{Li}_{8}\left[\left(\mathrm{CH}_{3}\right)_{2} \mathrm{NH}_{2}\right]_{4.5}\left[(\mathrm{PhPO})_{2} \mathrm{P}_{4} \mathrm{~W}_{24} \mathrm{O}_{92}\right] \cdot 35 \mathrm{H}_{2} \mathrm{O}$. 在该例化合物中，有机膦基团修饰于易发生反应的极 缺位, 这种结合方式阻碍了 $\left\{\mathrm{P}_{4} \mathrm{~W}_{24}\right\}$ 阴离子簇的后续反 应，使这种多缺位多酸得以结晶和进行单晶表征测试; 当然，在合成体系中加入过渡金属盐可使赤道缺位被 修饰, 同时相邻阴离子簇间可以通过 $\left\{\mathrm{CoO}_{6}\right\}$ 单元桥联, 形成沿 $a$ 方向伸展的无限一维链. 但值得注意的是, 该 化合物结构中同样存在缺位过渡金属离子与 $\mathrm{W}$ 之间的 无序, 故其分子式为: $\mathrm{H}_{2} \mathrm{~K}_{2} \mathrm{Li}_{1.5}\left[\left(\mathrm{CH}_{3}\right)_{2} \mathrm{NH}_{2}\right]_{4.5}\{[\mathrm{Co}$ $\left.\left.\left(\mathrm{H}_{2} \mathrm{O}\right)_{4}\right]_{2}\left[\left\{\left(\mathrm{H}_{2} \mathrm{O}\right)_{4} \mathrm{Co}\right\}(\mathrm{PhPO})_{2} \mathrm{P}_{4} \mathrm{~W}_{24} \mathrm{O}_{92}\right]\right\} \cdot 28 \mathrm{H}_{2} \mathrm{O} \quad\left(\left\{\mathbf{C o}_{3}\right.\right.$ $\left.(\mathbf{P h P O})_{2}\right\}$, 图4(e, f), 表1).

当二聚体中的桥联原子替换为主族金属离子时, 由于其离子半径相较过渡金属更大，二聚体间可通过
特殊的连接方式组装. 例如, 当所加入的杂原子合成

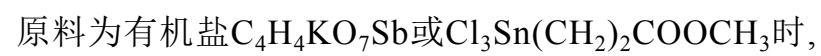
合成产物将会分别变为 $\mathrm{K}_{10}\left[\mathrm{H}_{6}\left\{\mathrm{P}_{2} \mathrm{~W}_{13} \mathrm{O}_{51}\right\}_{2} \mathrm{Sb}_{4}\right] \cdot 21 \mathrm{H}_{2} \mathrm{O}$ ( $\left\{\mathbf{S b}_{4}\right\}$, 图4(g), 表1)和 $\mathrm{Na}_{16}\left[\mathrm{~K}_{2} \mathrm{Na}_{2}\left\{\mathrm{Sn}\left(\mathrm{CH}_{2}\right)_{2} \mathrm{COO}\right\}_{4}\right.$ $\left.\left(\mathrm{H}_{2} \mathrm{O}\right)_{2}\left\{\mathrm{WO}_{5}\left(\mathrm{H}_{2} \mathrm{O}\right)\right\}\left(\mathrm{P}_{2} \mathrm{~W}_{15} \mathrm{O}_{56}\right)_{2}\right] \cdot 37 \mathrm{H}_{2} \mathrm{O} \quad\left(\left\{\mathbf{S n}_{4} \mathbf{W}\right\}\right.$, 图4

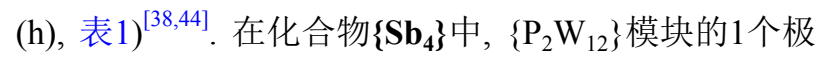
缺位被 $\left\{\mathrm{WO}_{6}\right\}$ 填充, 形成的 $\left\{\mathrm{P}_{2} \mathrm{~W}_{13}\right\}$ 首尾颠倒排列, 通 过 4 个三配位的 $\left\{\mathrm{SbO}_{3}\right\}$ 单元桥联; 而在 $\left\{\mathbf{S n}_{\mathbf{4}} \mathbf{W}\right\}$ 中, 与 $\mathrm{Sn}$ 配位连接的酯基螯合配体发生酸解, 4 个 $\left\{\mathrm{SnO}_{4} \mathrm{C}_{2}\right\}$ 单元 通过处于中心位置的 $\left\{\mathrm{WO}_{6}\right\}$ 桥联形成 $\left\{\mathrm{Sn}_{4} \mathrm{~W}\right\}$ 五元簇, 并作为桥联单元修饰于赤道缺位; 结构中剩余缺位被 6 个 $\left\{\mathrm{WO}_{6}\right\}$ 填充, 导致“类心型” $\left\{\mathrm{P}_{4} \mathrm{~W}_{24}\right\}$ 单元变形为开口 更大的“类船型”结构. 当然, 在 $\left\{\mathrm{P}_{2} \mathrm{~W}_{12}\right\}$ 模块簇合过程 中, 与锡配位的有机基团同样会影响最终产物的形成: 当所加杂原子原料为不含碳链的 $\left(\mathrm{CH}_{3}\right)_{2} \mathrm{SnCl}_{2}$ 时, $\mathrm{Sn}$ 原 子可与相邻铇氧簇通过 $\mathrm{O}$ 原子桥联, 两个甲基分布在 八面体对位, $\left\{\mathrm{P}_{4} \mathrm{~W}_{24}\right\}$ 单元在 $\left\{\mathrm{SnO}_{4} \mathrm{C}_{2}\right\}$ 的桥联下两两 垂直结合, 生成化合物 $\mathrm{K}_{17} \mathrm{Li}_{11}\left[\left\{\mathrm{Sn}\left(\mathrm{CH}_{3}\right)_{2}\right\}_{4}\left(\mathrm{H}_{2} \mathrm{P}_{4} \mathrm{~W}_{24}\right.\right.$ $\left.\left.\mathrm{O}_{92}\right)_{2}\right] \cdot 51 \mathrm{H}_{2} \mathrm{O}\left(\left\{\mathbf{S n}_{4}\right\} \text {, 图4(i), 表1 }\right)^{[44]}$. 当然, $\left\{\mathrm{P}_{2} \mathrm{~W}_{12}\right\}$ 的 二聚体化合物还包括之前内容提及的 $\left\{\mathbf{N b}_{\mathbf{1 2}}\right\}$, 在此不 再赘述. 


\section{$2.3\left\{\mathbf{P}_{2} \mathbf{W}_{12}\right\}$ 三聚体多酸化合物}

随着 $\left\{\mathrm{P}_{2} \mathrm{~W}_{12}\right\}$ 模块聚合数的增加, 其多聚体将会有 更多缺位位点可供修饰. 在三聚体系列中, 由于引入的 修饰单元主要扮演了桥联模块的角色，剩余空腔内需 存在若干数目的 $\mathrm{K}^{+}$平衡电荷以及通过静电引力稳定 阴离子簇结构. 例如, 在Kortz课题组 ${ }^{[39]}$ 报道的化合物 $\mathrm{K}_{6} \mathrm{Li}_{19}\left[\mathrm{Li}\left(\mathrm{H}_{2} \mathrm{O}\right) \mathrm{K}_{4}\left(\mathrm{H}_{2} \mathrm{O}\right)_{3}\left\{\left(\mathrm{UO}_{2}\right)_{4}\left(\mathrm{O}_{2}\right)_{4}\left(\mathrm{H}_{2} \mathrm{O}\right)_{2}\right\}_{2}\left(\mathrm{PO}_{3} \mathrm{OH}\right)_{2}-\right.$ $\left.\mathrm{P}_{6} \mathrm{~W}_{36} \mathrm{O}_{136}\right] \cdot 74 \mathrm{H}_{2} \mathrm{O}\left(\left\{\mathbf{U}_{8}\right\}\right.$, 图5(a), 表1)中, $\left\{\mathrm{UO}_{x}\right\}$ 单元均 分为两个四元方型环簇，作为桥联单元修饰于赤道缺 位上. 由于 $\mathrm{U}$ 离子所具有的高正电荷数和大离子半径, $\left\{\mathrm{P}_{6} \mathrm{~W}_{36}\right\}$ 单元伸展为类“缺位 $\left\{\mathrm{P}_{8} \mathrm{~W}_{48}\right\}$ ”的U型结构, 两 个 $\left\{\mathrm{PO}_{4}\right\}$ 修饰在剩余的两个极缺位上, 这种连接方式 与化合物 $\left\{\mathrm{Co}_{3}(\mathbf{P h P O})_{2}\right\}$ 类似. 除此之外，三个 $\left\{\mathrm{P}_{2} \mathrm{~W}_{12}\right\}$ 模块通常也可通过赤道位的桥联单元形成“肩并肩”的 结构，或通过极位的桥联单元形成“头接头”的环状 结构.

在“肩并肩”结构中, $\left\{\mathrm{P}_{2} \mathrm{~W}_{12}\right\}$ 的极缺位被 $\left\{\mathrm{WO}_{6}\right\}$ 填 充，赤道缺位则被间隔排布的 6 个 $\left\{\mathrm{WO}_{6}\right\}$ 和 6 个 $\left\{\mathrm{MO}_{6}\right\}$ 修饰, 由此生成结构通式为 $\left.\left\{\mathrm{P}_{2} \mathrm{~W}_{16} \mathrm{M}_{2} \mathrm{O}_{60}\right)_{3}\right\}^{n-}$ 的阴离子 簇 $\left(\left\{\mathbf{M}_{6}\right\}, \mathrm{M}=\mathrm{Mn}^{\mathrm{III}} 、 \mathrm{Fe}^{\mathrm{II}} 、 \mathrm{Co}^{\mathrm{III}}\right.$ 或 $\left.\mathrm{Ni}^{\mathrm{II}}\right)$. 在该类化合物的 研究中，王恩波团队 ${ }^{[66]}$ 将 $\left\{\mathrm{P}_{2} \mathrm{~W}_{12}\right\}$ 引入 $\mathrm{Mn}(\mathrm{OAc})_{2} / \mathrm{Ce}$ $\left(\mathrm{NO}_{3}\right)_{6}$ /乙酸盐的混合溶液中，成功合成一例含有铈锰 氧簇阳离子的三聚体化合物： $\mathrm{Na}_{20}\left[\mathrm{Ce}^{\mathrm{IV}}{ }_{3} \mathrm{Mn}^{\mathrm{IV}}{ }_{2} \mathrm{O}_{6}\right.$ $\left.(\mathrm{OAc})_{6}\left(\mathrm{H}_{2} \mathrm{O}\right)_{9}\right]_{2}\left[\mathrm{Mn}_{2}{ }_{2} \mathrm{P}_{2} \mathrm{~W}_{16} \mathrm{O}_{60}\right]_{3} \cdot 21 \mathrm{H}_{2} \mathrm{O} \quad\left(\left\{\mathbf{M n}_{6}\right\}\right.$, 图5 (b), 表 1); 而在Cronin团队 ${ }^{[40,41]}$ 的研究中, 使用 $\left\{\mathrm{P}_{2} \mathrm{~W}_{18}\right\}$ 作为合成原料, 可以在 Tris和 $\mathrm{TMCl}_{2}$ 的混合溶液中合成 三聚体．这种“粗粘”的合成方法可得到用 $\mathrm{Co} 、 \mathrm{Fe}$ 或Ni 修饰的同构阴离子簇 $\left(\right.$ 如 $\mathrm{Na}_{21} \mathrm{~K}_{2}\left[\mathrm{~K}\left(\mathrm{P}_{2} \mathrm{~W}_{16} \mathrm{Co}_{2} \mathrm{O}_{60}\right)_{3}\right]$ $\left.\cdot 73 \mathrm{H}_{2} \mathrm{O}\right)$, 却无法得到含有 $\left.\left\{\mathrm{P}_{2} \mathrm{~W}_{16} \mathrm{Mn}_{2} \mathrm{O}_{60}\right)_{3}\right\}^{n-}$ 的化合 物. 以上结果表明, 在复杂的酸性溶液系统中, 由 $\mathrm{Ce}^{4+}$ 和 $\mathrm{Mn}^{2+}$ 结合而成双雉状阳离子具有模板剂的作用, 可 以促进 $\left\{\mathbf{M n}_{\mathbf{6}}\right\}-$ 三聚体的形成.

在“头接头”的环状三聚结构中, $\left\{\mathrm{P}_{2} \mathrm{~W}_{12}\right\}$ 模块间通 过六配位的 $\left\{\mathrm{MO}_{6}\right\}$ 单元桥联成环; 空腔内的赤道缺位 可以作为活性位点, 以修饰若干数量的 $\mathrm{TM}$ 离子 ${ }^{[35,67]}$, 如 $\mathrm{Mn}^{2+} 、 \mathrm{Co}^{2+} 、 \mathrm{Ni}^{2+}$ 和 $\mathrm{Cu}^{2+}$. 另外, 在中心的六个位点 被修饰后, 三聚体还能继续与 $\mathrm{Gd}^{3+}$ 和有机配体连接, 形 成化合物 $\mathrm{K}_{3} \mathrm{Na}_{8}\left[\mathrm{~K}_{3} \subset\left\{\mathrm{GdMn}\left(\mathrm{H}_{2} \mathrm{O}\right)_{10}\right\}\left\{\mathrm{HMnGd}_{2}(\right.\right.$ Tart $) \mathrm{O}_{2}$ $\left.\left.\left(\mathrm{H}_{2} \mathrm{O}\right)_{15}\right\}\left\{\mathrm{P}_{6} \mathrm{~W}_{42} \mathrm{O}_{151}\left(\mathrm{H}_{2} \mathrm{O}\right)_{7}\right\}\right] \bullet 44 \mathrm{H}_{2} \mathrm{O}$ 和 $\mathrm{K}_{3} \mathrm{Na}_{10}\left[\mathrm{~K}_{3} \subset\right.$ $\left.\left\{\mathrm{GdCo}\left(\mathrm{H}_{2} \mathrm{O}\right)_{11}\right\}_{2}\left\{\mathrm{P}_{6} \mathrm{~W}_{41} \mathrm{O}_{148}\left(\mathrm{H}_{2} \mathrm{O}\right)_{7}\right\}\right] \cdot 43 \mathrm{H}_{2} \mathrm{O}\left(\left\{\mathbf{G d}_{\mathbf{3}} \mathbf{M n}_{2}\right\}\right.$ 和 $\left\{\mathbf{G d}_{2} \mathbf{C o}_{2}\right\}, \operatorname{Tart}=$ 酒石酸, 图5(e), 表 1$)^{[68]}$. 在两例化合 物中, $\left\{\mathrm{P}_{6} \mathrm{~W}_{39}\right\}$ 模块通过 $\left\{\mathrm{GdO}_{7}\right\}$ 单元桥联, 形成以无机 组分为主体的三维网络结构. 当然, 环状连接方式使三 聚体的环心可以修饰更多的过渡金属离子，此时空腔 内的碱金属离子数量缩减为 3 个. 例如, 在化合物 $\mathrm{K}_{12} \mathrm{Rb}_{3}\left[\mathrm{Rb}_{33}\left\{\mathrm{~V}^{\mathrm{V}} \mathrm{V}^{\mathrm{IV}}{ }_{3} \mathrm{O}_{7}\left(\mathrm{H}_{2} \mathrm{O}\right)_{6}\right\}_{2}\left\{\left(\mathrm{H}_{2} \mathrm{P}_{2} \mathrm{~W}_{12} \mathrm{O}_{48}\right)_{3}(\mathrm{WO}-\right.\right.$ $\left.\left.\left.\left(\mathrm{H}_{2} \mathrm{O}\right)\right)_{3}\right\}\right] \cdot 63 \mathrm{H}_{2} \mathrm{O}\left(\left\{\mathbf{V}_{\mathbf{8}} \mathbf{W}_{3}\right\}\right.$, 图5(d), 表1)中, $\mathrm{V}^{\mathrm{IV}}$ 与 $\mathrm{O}$ 原子 配位形成的 $\left\{\mathrm{VO}_{6}\right\}$ 八面体桥联了相邻的 $\left\{\mathrm{P}_{2} \mathrm{~W}_{12}\right\}$ 模块,
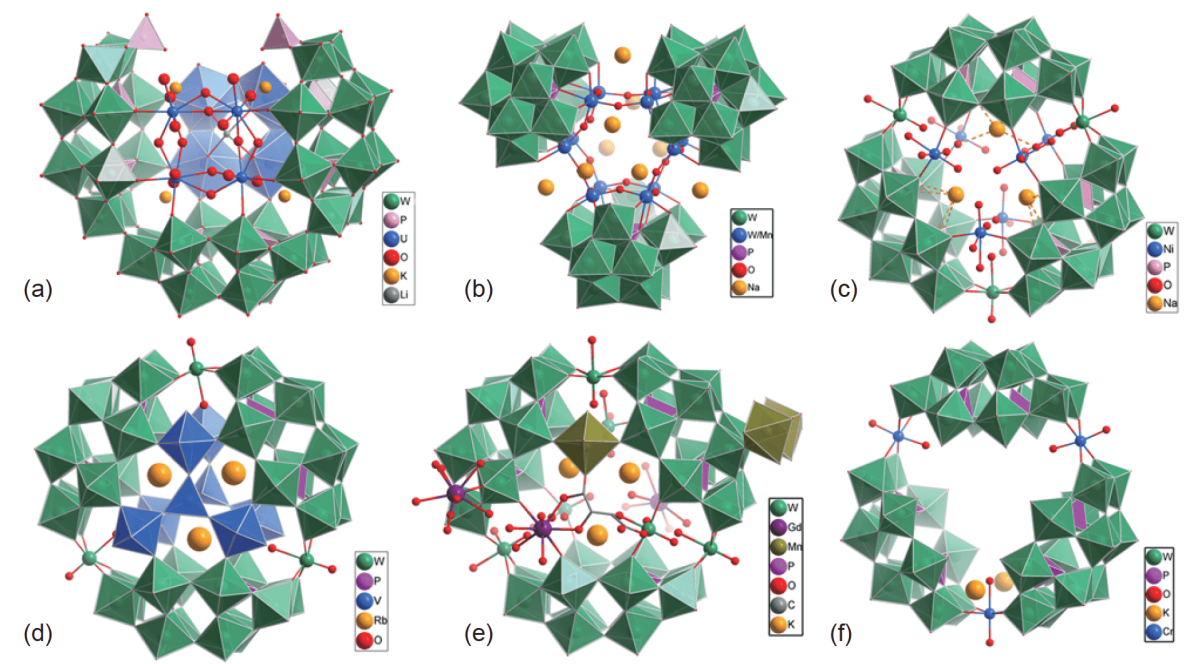

图 5 (a f) 不同金属(按顺序依次为 $\left\{\mathbf{U}_{\mathbf{8}}\right\} 、\left\{\mathbf{M n}_{\mathbf{6}}\right\} 、\left\{\mathbf{N i}_{\mathbf{6}} \mathbf{W}_{3}\right\} 、\left\{\mathbf{V}_{\mathbf{8}}\right\} 、\left\{\mathbf{G d}_{\mathbf{3}} \mathbf{M n}_{\mathbf{2}}\right\}$ 和 $\left\{\mathbf{C r}_{\mathbf{3}}\right\}$ )修饰的 $\left\{\mathrm{P}_{2} \mathrm{~W}_{12}\right\}$ 三聚体基多酸化合物结 构示意图(网络版彩图)

Figure 5 (a-f) Structural illustration of different metal $\left(\left\{\mathbf{U}_{\mathbf{8}}\right\},\left\{\mathbf{M n}_{6}\right\},\left\{\mathbf{N i}_{\mathbf{6}} \mathbf{W}_{3}\right\},\left\{\mathbf{V}_{\mathbf{8}}\right\},\left\{\mathbf{G d}_{3} \mathbf{M n}_{2}\right\}\right.$, and $\left\{\mathbf{C r}_{3}\right\}$, respectively) modified $\left\{\mathrm{P}_{2} \mathrm{~W}_{12}\right\}$ trimerbased POMs (color online). 
而 $\mathrm{V}^{\mathrm{V}}$ 与 $\mathrm{O}$ 原子配位形成的 $\left\{\mathrm{VO}_{4}\right\}$ 用于连接三个 $\left\{\mathrm{VO}_{6}\right\}$ 单元, 两个 $\mathrm{Y}$ 字形的 $\left\{\mathrm{V}_{4}\right\}$ 簇封于空腔首尾, 空腔内存在 3 个 $\mathrm{Rb}^{+}$离子以保证骨架的稳定 ${ }^{[42]}$; 而当修饰单元换为 离子半径较大的 $\mathrm{Ni}^{2+}$ 时, $\left\{\mathrm{NiO}_{6}\right\}$ 单元间不存在直接桥 联单元，这种修饰方式压缩了空腔的体积，所以空腔 内的碱金属离子便变为离子半径略小的 $\mathrm{K}^{+}$(如化合物 $\left\{\mathbf{N i}_{6} \mathbf{W}_{3}\right\}$, 图5(c), 表1).

\section{$2.4\left\{\mathrm{P}_{2} \mathrm{~W}_{12}\right\}$ 四聚体及 $\left\{\mathrm{P}_{8} \mathrm{~W}_{48}\right\}$ 基多酸化合物}

在酸性条件下, $\left\{\mathrm{P}_{2} \mathrm{~W}_{12}\right\}$ 模块易发生自组装, 形成 环状的 $\left[\mathrm{H}_{7} \mathrm{P}_{8} \mathrm{~W}_{48} \mathrm{O}_{184}\right]^{33-}$ 单元, 由于其具有较高的结构 稳定性和产率及较大的中心空腔(直径约为 $10 \AA$ ), 可 作为合成多核金属团簇的模板，因此受到研究者的青 睐。不过，也正是因为“易自组装”这一特性，不含 $\left\{\mathrm{P}_{8} \mathrm{~W}_{48}\right\}$ 单元的 $\left\{\mathrm{P}_{2} \mathrm{~W}_{12}\right\}$ 四聚体化合物数量有限, 除前 文提及的 $\left\{\mathbf{N b}_{28}\right\} 、\left\{\mathrm{Nb}_{32}\right\}$ 和 $\left\{\mathbf{S n}_{4}\right\}$ 外，仅有 $\left\{\mathrm{Fe}_{28}\right\}$ 和 $\left\{\mathbf{M n}_{16}\right\}$ 两例化合物(图6(a, b), 表1 $)^{[32,45]}$. 化合物 $\left\{\mathbf{F e}_{28}\right\}$ 阴离子簇的结合方式类似于 $\left\{\mathbf{S n}_{4}\right\},\left\{\mathrm{P}_{2} \mathrm{~W}_{12}\right\}$ 模块在 10 个 $\left\{\mathrm{FeO}_{6}\right\}$ 补位单元和两个 $\left\{\mathrm{FeO}_{6}\right\}$ 桥联单元的连接下发 生二聚，继而两个二聚体间又通过 4 个 $\left\{\mathrm{FeO}_{6}\right\}$ 单元桥 联，沿垂直方向相互组装. 化合物 $\left\{\mathbf{M n}_{16}\right\}$ 则是六聚体 化合物 $\left\{\mathbf{M n}_{24}\right\}$ 的重组产物，其阴离子簇由 4 个 $\left\{\gamma-\mathrm{P}_{2} \mathrm{~W}_{12^{-}}\right.$ $\left.\mathrm{O}_{48} \mathrm{Mn}_{4}\left(\mathrm{H}_{2} \mathrm{O}\right)_{6}\right\}^{6-}$ 单元组装而成. 在该单元中, $\left\{\mathrm{P}_{2} \mathrm{~W}_{12}\right\}$ 的极缺位被两个 $\left\{\mathrm{MnO}_{6}\right\}$ 单元修饰，赤道缺位被 $\left\{\mathrm{Mn}_{2}\right.$ $\left.\mathrm{O}_{10}\right\}$ 单元修饰, 相邻单元通过 $\left\{\mathrm{Mn}_{2} \mathrm{O}_{10}\right\}$ 与极位 $\left\{\mathrm{WO}_{6}\right\}$ 的桥联实现组装, 形成扭曲的环状四聚体结构 ( $\left\{\mathrm{P}_{8} \mathrm{~W}_{48}\right\}$ 基化合物归纳总结见图7).

使用 $\left\{\mathrm{P}_{8} \mathrm{~W}_{48}\right\}$ 作为模板进行多酸合成的相关研究 起始于Kortz研究组. 在该研究组2004年的系列报道中, $\left\{\mathrm{P}_{8} \mathrm{~W}_{48}\right\}$ 模块可以在加热条件下与过渡金属离子结合, 生成具有较高核数的化合物, 如 $\mathrm{K}_{16} \mathrm{Li}_{11}\left[\left\{\mathrm{~K}\left(\mathrm{H}_{2} \mathrm{O}\right)\right\}\{\mathrm{Ru}-\right.$ (p-cymene) $\left.\left.\left(\mathrm{H}_{2} \mathrm{O}\right)\right\}_{4} \mathrm{P}_{8} \mathrm{~W}_{49} \mathrm{O}_{186}\left(\mathrm{H}_{2} \mathrm{O}\right)_{2}\right] \cdot 87 \mathrm{H}_{2} \mathrm{O} \quad\left(\left\{\mathbf{R u}_{4}\right\}\right)$ 和 $\mathrm{K}_{12} \mathrm{Li}_{13}\left[\mathrm{Cu}_{20} \mathrm{Cl}(\mathrm{OH})_{24}\left(\mathrm{H}_{2} \mathrm{O}\right)_{12}\left(\mathrm{P}_{8} \mathrm{~W}_{48} \mathrm{O}_{184}\right)\right] \cdot 22 \mathrm{H}_{2} \mathrm{O}$

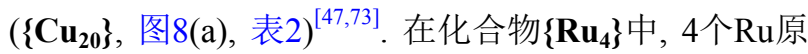
子位于同一平面上, 另有 4 个 $\mathrm{K}$ 原子分布在其他空腔内, 由于这种特殊的分布方式，阴离子簇中的电荷分布变 得不均匀，进而导致 $\left\{\mathrm{P}_{8} \mathrm{~W}_{48}\right\}$ 骨架产生形变(原尺寸约 $20.3 \AA \times 20.3 \AA$ ，现尺寸约 $19.5 \AA \times 21.0 \AA$ ); 而在化合物 $\left\{\mathbf{C u}_{20}\right\}$ 中，骨架内部的 20 个 $\mathrm{Cu}$ 原子具有多种配位构型, 可分为以下三类：用于桥联相邻 $\left\{\mathrm{P}_{2} \mathrm{~W}_{12}\right\}$ 的 8 个 $\left\{\mathrm{CuO}_{6}\right\}$ 单元(蓝色球标识)、桥联 $c$ 方向上相邻 $\left\{\mathrm{CuO}_{6}\right\}$ 的 4 个

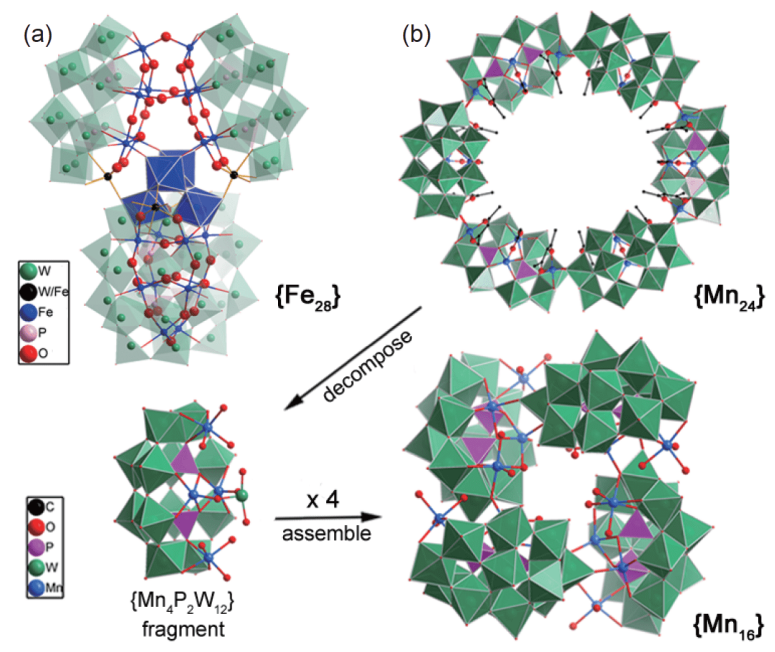

图 6 (a, b) 过渡金属 $\left\{\mathrm{Fe}_{28}\right\} 、\left\{\mathrm{Mn}_{16}\right\}$ 和 $\left\{\mathrm{Mn}_{24}\right\}$ 修饰的 $\left\{\mathrm{P}_{2} \mathrm{~W}_{12}\right\}$ 四聚体/六聚体基多酸化合物结构示意图(网络版彩 图)

Figure $6(\mathrm{a}, \mathrm{b})$ Structural illustration of transition metal $\left(\left\{\mathrm{Fe}_{28}\right\}\right.$ and $\left.\left\{\mathbf{M n}_{24}\right\} /\left\{\mathbf{M n}_{16}\right\}\right)$ modified $\left\{\mathrm{P}_{2} \mathrm{~W}_{12}\right\}$ tetramer- or hexamer-based POMs (color online).

$\left\{\mathrm{CuO}_{5}\right\}$ 单元(橙色四棱雉标识), 以及剩余的 8 个 $\left\{\mathrm{CuO}_{4}\right\}$ 单元(黑色球标识), $\mathrm{Cu}^{2+}$ 灵活的配位方式在这一结构中 得以体现. 有趣的是, Nadjo研究组 ${ }^{[48]}$ 也报道了一例类 似化合物，其 $\left\{\mathrm{P}_{8} \mathrm{~W}_{48}\right\}$ 单元同样被 20 个 $\mathrm{Cu}^{2+}$ 修饰: 由于 合成过程中加入的少量的 $\mathrm{NaN}_{3}$, 多酸骨架被叠氮基团 修饰，形成了分子式为 $\mathrm{LiK}_{14} \mathrm{Na}_{9}\left[\mathrm{P}_{8} \mathrm{~W}_{48} \mathrm{O}_{184} \mathrm{Cu}_{20}\left(\mathrm{~N}_{3}\right)_{6}\right.$ $\left.(\mathrm{OH})_{18}\right] \cdot 60 \mathrm{H}_{2} \mathrm{O} \quad\left(\left\{\mathbf{C u}_{20}\right.\right.$-azido $\}$, 图8(b), 表2)的化合物. 同样，阴离子簇的 20 个 $\mathrm{Cu}$ 原子可分为两类: 以扭曲八 面体构型存在, 用于桥联相邻 $\left\{\mathrm{P}_{2} \mathrm{~W}_{12}\right\}$ 单元的 4 个 $\left\{\mathrm{CuO}_{6}\right\}$; 以及以三棱双锥体构型存在, 填充在 $\left\{\mathrm{P}_{2} \mathrm{~W}_{12}\right\}$ 赤道位的 16 个 $\left\{\mathrm{CuO}_{5}\right\}$. 根据两例化合物合成条件与结 构的不同, 他们分析其原因可能是: 空腔内带有负电荷 的 $\mathrm{Cl}^{-}$合成过程中起到了模板剂的作用，而在 $\left\{\mathbf{C u}_{20}{ }^{-}\right.$ azido $\}$ 的合成中, $\mathrm{CuCl}_{2}$ 被替换为 $\mathrm{Cu}\left(\mathrm{CH}_{3} \mathrm{COO}\right)_{2}$; 其次, 该化合物中与 $\mathrm{Cu}(\mathrm{II})$ 中心连接的羟基不能够被叠氮基 所取代.

同年, Müller课题组 ${ }^{[50]}$ 报道了与 $\left\{\mathbf{R u}_{4}\right\}$ 的阴离子 结构类似的化合物，其分子式为 $\mathrm{Na}_{12} \mathrm{~K}_{8} \mathrm{H}_{4}\left[\mathrm{~K}_{8}\left\{\mathrm{P}_{8} \mathrm{~W}_{48}\right.\right.$ $\left.\left.\left.\mathrm{O}_{184}\right\}\right]\left\{\mathrm{V}_{4}^{\mathrm{V}} \mathrm{V}^{\mathrm{IV}}{ }_{2} \mathrm{O}_{12}\left(\mathrm{H}_{2} \mathrm{O}\right)_{2}\right\}_{2}\right]_{2} \cdot 80 \mathrm{H}_{2} \mathrm{O}\left(\left\{\mathbf{V}_{12}\right\}\right)$. 由于合成 过程中使用 $\mathrm{VOSO}_{4}$ 作为钒源, 离子半径更大的 $\mathrm{V}(\mathrm{IV})$ 就 能够与更多 $\mathrm{O}$ 原子配位, 形成用于桥联相邻 $\left\{\mathrm{P}_{2} \mathrm{~W}_{12}\right\}$ 单 元的 $\left\{\mathrm{V}^{\mathrm{IV}} \mathrm{O}_{6}\right\}$; 氧化后的 $\mathrm{V}(\mathrm{V})$ 成键能力较弱, 形成的 


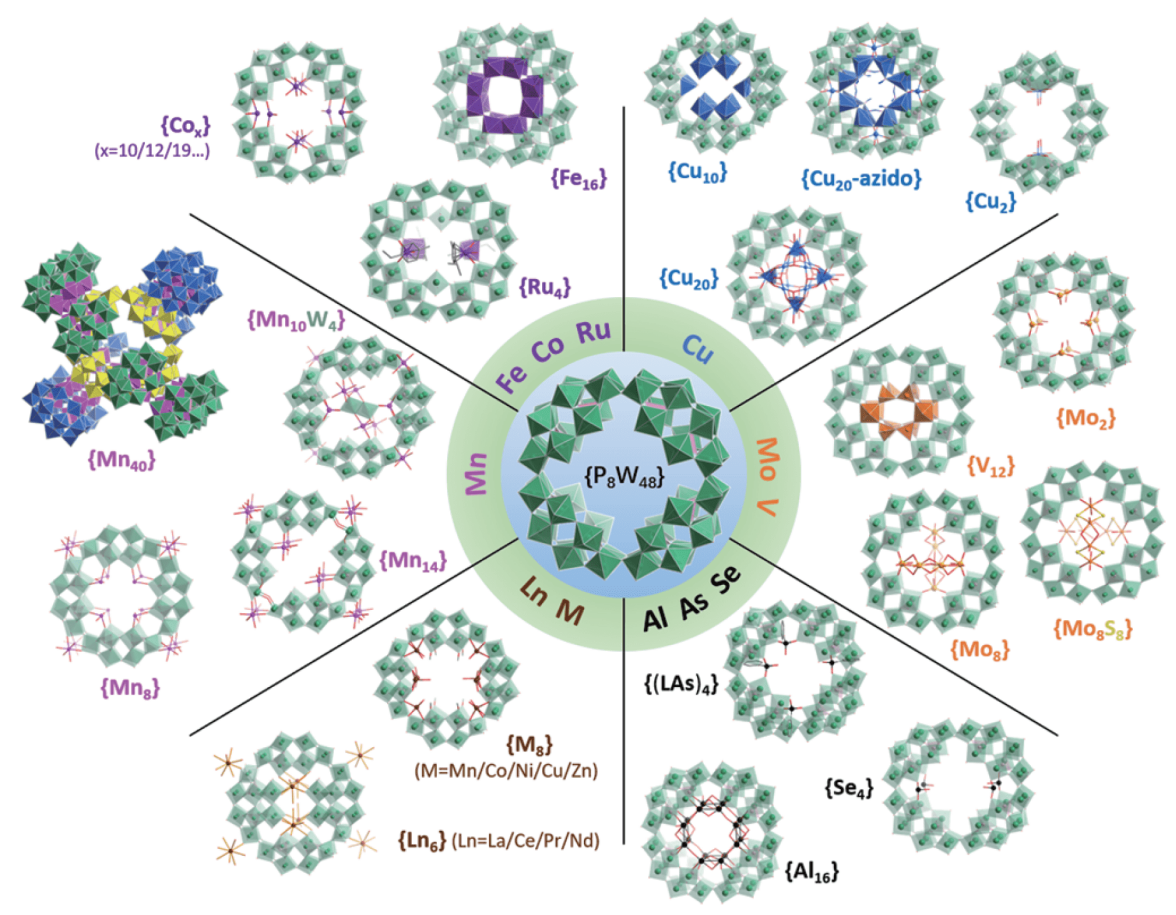

图 7 不同元素修饰的 $\left\{\mathrm{P}_{8} \mathrm{~W}_{48}\right\}$ 基多酸化合物汇总图(网络版彩图)

Figure 7 Summary of different element modified $\left\{\mathrm{P}_{8} \mathrm{~W}_{48}\right\}$-based POMs (color online).
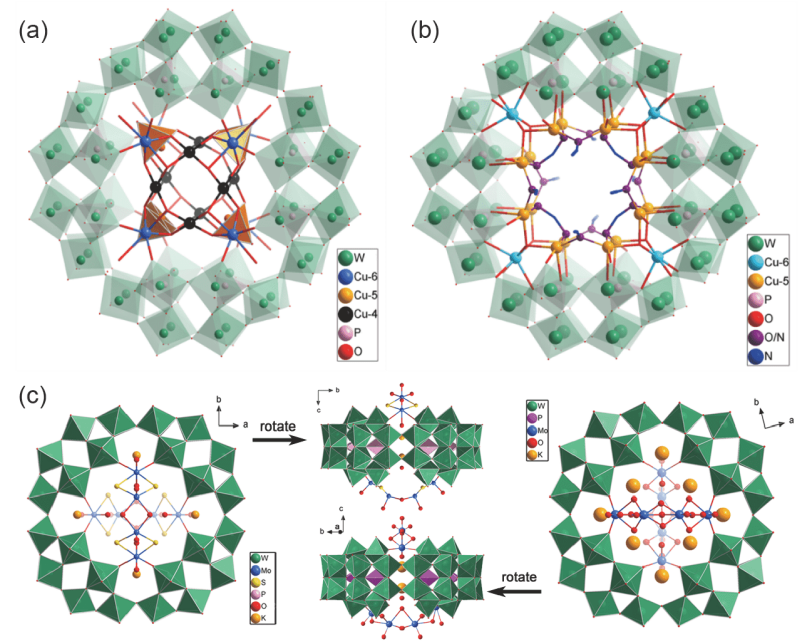

图 8 (a) $\left\{\mathrm{Cu}_{20}\right\} 、$ (b) $\left\{\mathrm{Cu}_{20}\right.$-azido $\} 、$ (c) $\left\{\mathrm{Mo}_{8} \mathrm{~S}_{8}\right\}$ 和 $\left\{\mathrm{Mo}_{12}\right\}$ 修 饰的 $\left\{\mathrm{P}_{8} \mathrm{~W}_{48}\right\}$ 基聚阴离子结构示意图(网络版彩图)

Figure 8 Structural illustration of (a) $\left\{\mathrm{Cu}_{20}\right\}$, (b) $\left\{\mathrm{Cu}_{20}\right.$-azido $\}$, and (c) $\left\{\mathbf{M o}_{8} \mathrm{~S}_{8}\right\}$ and $\left\{\mathbf{M o}_{12}\right\}$-modified $\left\{\mathrm{P}_{8} \mathrm{~W}_{48}\right\}$-based polyoxoanions (color online).

$\left\{\mathrm{V}^{\mathrm{V}} \mathrm{O}_{4}\right\}$ 单元需要两两连接才能桥联相邻的 $\left\{\mathrm{P}_{2} \mathrm{~W}_{12}\right\}$ 单 元. 除此之外, 骨架内的空腔被 8 个 $\mathrm{K}^{+}$填充, 这样的连接 方式使多酸骨架变形为 $19.1 \AA \times 21.5 \AA$ 的类椭圆形. 继
而, 该研究组又报道了一例由 $\mathrm{Fe}$ 原子修饰的 $\left\{\mathrm{P}_{8} \mathrm{~W}_{48}\right\}$ 结 构: $\mathrm{Na}_{9} \mathrm{~K}_{11}\left[\mathrm{P}_{8} \mathrm{~W}_{48} \mathrm{O}_{184} \mathrm{Fe}_{16}(\mathrm{OH})_{28}\left(\mathrm{H}_{2} \mathrm{O}\right)_{4}\right] \cdot 100 \mathrm{H}_{2} \mathrm{O}$ $\left(\left\{\mathrm{Fe}_{16}\right\}\right)^{[56]}$. 在该化合物中, 16 个 Fe原子均匀地以 $\left\{\mathrm{FeO}_{6}\right\}$ 形式填充在 4 个 $\left\{\mathrm{P}_{2} \mathrm{~W}_{12}\right\}$ 单元的赤道位, 相邻的 $\left\{\mathrm{P}_{2} \mathrm{~W}_{12}\right\}$ 单元各提供一个 $\left\{\mathrm{FeO}_{6}\right\}$, 使两个八面体以共边 的方式桥联; 而来自相同 $\left\{\mathrm{P}_{2} \mathrm{~W}_{12}\right\}$ 单元的两个 $\left\{\mathrm{FeO}_{6}\right\}$ 单 元通过 $\mu_{2}$-O连接(共角). 同时在该篇报道中, 他们还分 别尝试了使用 $\mathrm{FeCl}_{3} \cdot 6 \mathrm{H}_{2} \mathrm{O} 、 \mathrm{Fe}\left(\mathrm{ClO}_{4}\right)_{3} \cdot x \mathrm{H}_{2} \mathrm{O} 、 \mathrm{FeSO}_{4}$ ・ $7 \mathrm{H}_{2} \mathrm{O} 、\left[\mathrm{Fe}_{3} \mathrm{O}(\mathrm{Ac})_{6}\left(\mathrm{H}_{2} \mathrm{O}\right)_{3}\right] \mathrm{Cl} \bullet \mathrm{H}_{2} \mathrm{O}$ 和 $\mathrm{FeCl}_{2} \cdot 4 \mathrm{H}_{2} \mathrm{O}$ 五种含 $\mathrm{Fe}$ 原料进行晶体合成, 结果显示除合成产物的碱金属 离子 $\left(\mathrm{K}^{+} 、 \mathrm{Li}^{+} / \mathrm{Na}^{+}\right)$含量有所不同外, 使用 $\mathrm{FeCl}_{3} \cdot 6 \mathrm{H}_{2} \mathrm{O}$ 作为Fe源的合成路径产物产率更高.

除了使用 $\mathrm{Nb}$ 或 $\mathrm{V}$ 对 $\left\{\mathrm{P}_{2} \mathrm{~W}_{12}\right\}$ 进行缺位修饰, 同属于 附加原子的Mo也是一种可行选择. Müller研究组 ${ }^{[1]}$ 与 Cadot研究组 ${ }^{[52]}$ 分别使用 $\mathrm{Na}_{2} \mathrm{MoO}_{4} \cdot 2 \mathrm{H}_{2} \mathrm{O}$ 和 $\mathrm{K}_{2-x}\left(\mathrm{NMe}_{4}\right)_{x^{-}}$ $\left[\mathrm{I}_{2} \mathrm{Mo}_{10} \mathrm{O}_{10} \mathrm{~S}_{10}(\mathrm{OH})_{10}\left(\mathrm{H}_{2} \mathrm{O}\right)_{5}\right] \cdot 20 \mathrm{H}_{2} \mathrm{O}$ 作为Mo源, 合成了 $\mathrm{K}_{10} \mathrm{Na}_{14}\left[\mathrm{~K}_{8}\left(\mathrm{P}_{8} \mathrm{~W}_{48} \mathrm{O}_{184}\right)\left(\mathrm{Mo}^{\mathrm{VI}} \mathrm{O}_{2}\right)_{4}\left(\mathrm{Mo}_{4}{ }_{4} \mathrm{O}_{13}\right)_{2}\right] \cdot 80 \mathrm{H}_{2} \mathrm{O}$ $\left(\left\{\mathbf{M o}_{12}\right\}\right)$ 和 $\mathrm{K}_{20} \mathrm{Li}_{6} \mathrm{H}_{4}\left[\mathrm{~K}_{4}\left\{\mathrm{Mo}_{4} \mathrm{O}_{4} \mathrm{~S}_{4}(\mathrm{OH})_{2}\left(\mathrm{H}_{2} \mathrm{O}\right)_{3}\right\}_{2}\left(\mathrm{WO}_{2}\right)-\right.$ $\left.\left(\mathrm{P}_{8} \mathrm{~W}_{48} \mathrm{O}_{184}\right)\right] \cdot 95 \mathrm{H}_{2} \mathrm{O}\left(\left\{\mathbf{M o}_{8} \mathbf{S}_{8}\right\}\right)$ 两种化合物(图8(c), 表 2). 从局部视角看, 两种阴离子中都存在异面交叉的 
$\left\{\mathrm{Mo}_{4}\right\}$ 链结构, 其位于 $\left\{\mathrm{P}_{8} \mathrm{~W}_{48}\right\}$ 两侧, 起到桥联作用. 不 同的是, 在化合物 $\left\{\mathrm{Mo}_{12}\right\}$ 中, 两条 $\left\{\mathrm{Mo}_{4}\right\}$ 链中的Mo均以 +5 价形式存在，相对更大的离子半径使其能够与半径 较小的氧原子配位; 其余 4 个 $\mathrm{Mo}^{\mathrm{VI}}$ 分布在剩余的桥联 空位中, $\mathrm{Mo}^{\mathrm{VI}}$ 的端氧与 $\left\{\mathrm{Mo}_{4}\right.$ 链 $\}$ 的 $\mu_{2}-\mathrm{O}$ 存在氢键作用, 结构中混合价态的Mo离子决定了化合物的合成条件 是苛刻的(摚拌、加热及冷却液的结晶过程均需要使 用 $\mathrm{N}_{2}$ 作为保护气). 在化合物 $\left\{\mathbf{M o}_{8} \mathbf{S}_{\mathbf{8}}\right\}$ 中, 成键半径较大 的 $\mathrm{S}$ 原子能够与 $\mathrm{Mo}$ 原子形成更长的 $\left\{\mathrm{Mo}_{4}\right\}$ 链，剩余的 4 个桥联空位被 $\mathrm{K}^{+}$填充. 另外，由于合成过程使用了硫 钼碘酸的四甲基铵盐，需加入过量的 $\mathrm{NaBPh}_{4}$ 以除去影 响原料溶解的 $\mathrm{K}^{+}$. 在王恩波团队 ${ }^{[70]}$ 的类似报道中，他 们将原料 $\left\{\mathrm{P}_{8} \mathrm{~W}_{48}\right\}$ 溶解在 $\left[\mathrm{Mo}_{2} \mathrm{O}_{4}\left(\mathrm{H}_{2} \mathrm{O}\right)_{6}\right]^{2+}$ 溶液中, 设置 了体系加入 $\mathrm{CuCl}_{2} \cdot 2 \mathrm{H}_{2} \mathrm{O}$ 与否的对照实验，成功合成化 合物: $\mathrm{Na}_{12} \mathrm{Li}_{16}\left\{\left[\mathrm{Cu}\left(\mathrm{H}_{2} \mathrm{O}\right)\right]_{2}\left[\mathrm{Cu}_{4}(\mathrm{OH})_{4}\left(\mathrm{H}_{2} \mathrm{O}\right)_{8}\right]_{2} \mathrm{P}_{8} \mathrm{~W}_{48^{-}}\right.$ $\left.\mathrm{O}_{184}\right\} \cdot 55 \mathrm{H}_{2} \mathrm{O} \quad\left(\left\{\mathbf{C u}_{10}\right\}\right)$ 和 $\mathrm{K}_{4} \mathrm{Na}_{24} \mathrm{Li}_{10}\left\{\left(\mathrm{MoO}_{2}\right)_{2}\left(\mathrm{P}_{8} \mathrm{~W}_{48}\right.\right.$ $\left.\left.\mathrm{O}_{184}\right)\right\} \bullet 61 \mathrm{H}_{2} \mathrm{O}\left(\left\{\mathbf{M o}_{2}\right\}\right)$. 在 $\left\{\mathbf{C u}_{10}\right\}$ 中, 位于 $\left\{\mathrm{P}_{2} \mathrm{~W}_{12}\right\}$ 极位 的桥联单元均为六配位的 $\mathrm{Li}^{+}, 8$ 个 $\left\{\mathrm{CuO}_{6}\right\}$ 四四成组填 充相对 $\left\{\mathrm{P}_{2} \mathrm{~W}_{12}\right\}$ 单元的赤道位，剩余两个 $\left\{\mathrm{CuO}_{5}\right\}$ 单元 存在无序，填充在赤道位中心; 而在 $\left\{\mathrm{Mo}_{2}\right\}$ 中，两个Mo 原子无序地分布在 8 个位置上, 在与两个端氧配位的同 时，与相邻的 $\left\{\mathrm{WO}_{6}\right\}$ 通过 $\mu_{2}-\mathrm{O}$ 连接，起到桥联相邻 $\left\{\mathrm{P}_{2} \mathrm{~W}_{12}\right\}$ 单元的作用. 值得注意的是，尽管化合物 $\left\{\mathbf{C u}_{\mathbf{1 0}}\right\}$ 中不含 $\mathrm{Mo}$, 但若合成过程中不加入 $\left[\mathrm{Mo}_{2} \mathrm{O}_{4}\right.$ $\left.\left(\mathrm{H}_{2} \mathrm{O}\right)_{6}\right]^{2+}$, 将会使产物变为单一的 $\left\{\mathbf{C u}_{20}\right\}$; 另外，合成 体系中加入的 $\mathrm{Cu}^{2+}$ 也会使 $\left\{\mathrm{P}_{8} \mathrm{~W}_{48}\right\}$ 无法与 $\mathrm{Mo}$ 配位.
使用 $\mathrm{Mn} 、 \mathrm{Co}$ 或 $\mathrm{Ln}$ 对 $\left\{\mathrm{P}_{8} \mathrm{~W}_{48}\right\}$ 模块进行修饰，则可 实现 $\left\{\mathrm{P}_{8} \mathrm{~W}_{48}\right\}$ 单元间的相互连接，形成POM纳米立方 体或具有孔道的无机网络. 例如, $\left\{\mathrm{P}_{8} \mathrm{~W}_{48}\right\}$ 单元通过 $\mathrm{Mn}^{2+}$ 连接所形成，具有独特空腔结构的“立方族 状”\{ $\left\{\mathbf{M n}_{\mathbf{8}}\right\}$ 和“筒状”\{ $\left\{\mathbf{M n}_{\mathbf{1 4}}\right\}$ (图9(a, b)，表2). 对于 $\left\{\mathbf{M n}_{\mathbf{8}}\right\}$ 聚阴离子而言, 六个 $\left\{\mathrm{P}_{8} \mathrm{~W}_{48}\right\}$ 模块间通过 $\mathrm{Mn}^{\mathrm{II}}$ 桥联实现 “面向面”自组装，这种组合方式使立方体中心存在约 $7.24 \mathrm{~nm}^{3}$ 体积的空腔 ${ }^{[33]}$. 通过控制桥 $\mathrm{Mn}$ 离子氧化态以 调控骨架所带的负电荷数，进而可实现空腔内外碱金 属离子与 TM离子的交换. 离子交换实验结果显示, 每 克该化合物可以在 $24 \mathrm{~h}$ 内吸收 $0.73 \mathrm{mmol}$ 的 $\mathrm{Cu}^{\mathrm{II}}$ (约对应 于每个八面体单元吸收了 35 个 $\mathrm{Cu}^{\mathrm{II}}$ )，且多酸骨架结构 可在离子交换过程中保持稳定，这一研究表明具有中 心空腔的环状 $\left\{\mathrm{P}_{8} \mathrm{~W}_{48}\right\}$ 是一种稳定的构筑模块, 可以用 于功能性无机框架的设计; 在Co或Ln修饰的系列化合 物中, $\left\{\mathrm{P}_{8} \mathrm{~W}_{48}\right\}$ 单元会根据修饰数量或位置的变化, 以 不同方式相互堆叠形成无机三维网络结构(图9(c), 表 2 ); 同时, $\left\{\mathbf{C o}_{x}\right\}$ 系列化合物的光催化析氢活性也会根 据 $\mathrm{Co}^{2+}$ 修饰数量和位置的不同而有所改变 $($ 相同实验 条件下，\{ $\left\{\mathbf{C o}_{10.5}\right\} 、\left\{\mathbf{C o}_{12}\right\}$ 和 $\left\{\mathbf{C o}_{\mathbf{1 9}}\right\}$ 分别为418.3、346.7 和1385 $\mu \mathrm{mol} \mathrm{h} \mathrm{g}^{-1}$ ).

除上述连接方式外，在Kögerler研究组 ${ }^{[55]}$ 的报道

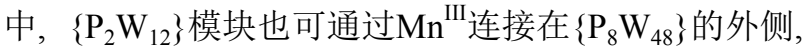
形成单分子包含 40 个 $\mathrm{Mn}^{\mathrm{III}}$ 的高核聚阴离子化合物: $\mathrm{K}_{56} \mathrm{Li}_{74} \mathrm{H}_{141}\left[\left(\mathrm{P}_{8} \mathrm{~W}_{48} \mathrm{O}_{184}\right)\left\{\left(\mathrm{Mn}_{4} \mathrm{P}_{2} \mathrm{~W}_{14} \mathrm{O}_{60}\right)\left(\mathrm{Mn}_{3} \mathrm{P}_{2} \mathrm{~W}_{15} \mathrm{O}_{58}\right)_{2}\right\}_{4}\right]$ -680 $\mathrm{H}_{2} \mathrm{O}\left(\left\{\mathbf{M n}_{40}\right\}\right.$, 图9(d), 表2). 在该化合物中, 12 个 (a)

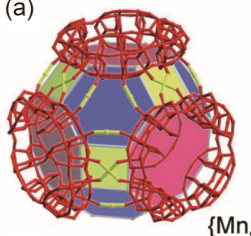

(b)

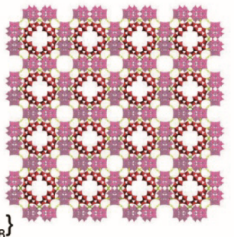

(c)
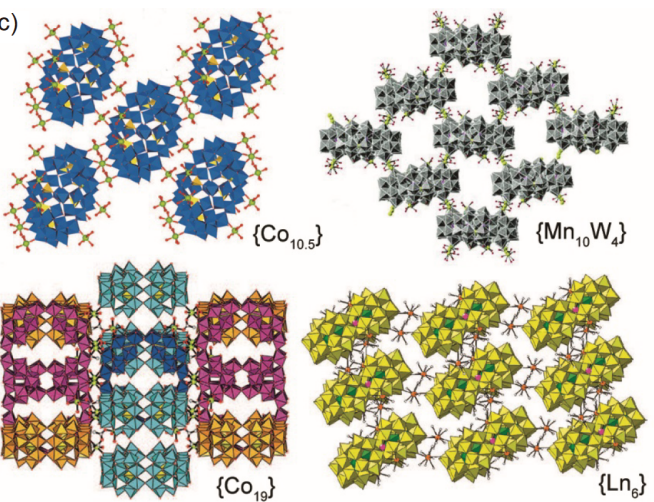

(d)

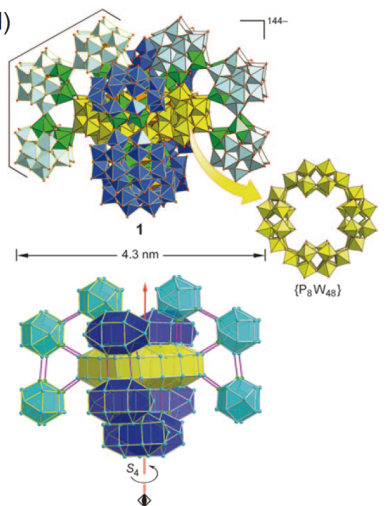

图 9 (a) $\left\{\mathbf{M n}_{\mathbf{8}}\right\}$ 修饰的聚阴离子“立方簇”结构及其三维堆叠图 ${ }^{[53]}$; (b) $\left\{\mathbf{M n}_{\mathbf{1 4}}\right\}$ 修饰的聚阴离子“筒状”结构，及其 $b$ 方向视角 ${ }^{[54]}$; (c) $\left\{\mathrm{P}_{8} \mathrm{~W}_{48}\right\}$ 单元通过 $\mathrm{Co}^{2+} / \mathrm{Mn}^{2+} / \mathrm{Ln}^{3+}$ 连接形成的多种无机三维网络结构 ${ }^{[71,72,76]} ;(\mathrm{d})\left\{\mathbf{M n}_{40}\right\}$ 修饰的阴离子等结构及其简化示意 图 $^{[55]}$ (网络版彩图)

Figure 9 (a) The cubic cluster formed by $\left\{\mathbf{M n}_{\mathbf{8}}\right\}$-modified polyanions, and corresponding 3D packing structure [53]; (b) the barrel structure formed by $\left\{\mathbf{M n}_{14}\right\}$-modified polyanions and the view along $b$ direction [54]; (c) various inorganic $3 \mathrm{D}$ networks formed by $\left\{\mathrm{P}_{8} \mathrm{~W}_{48}\right\}$ units and $\mathrm{Co}^{2+} / \mathrm{Mn}^{2+} / \mathrm{Ln}^{3+}$ ions [71,72,76]; (d) the polyhedral structure and simplified diagram of $\left\{\mathbf{M n}_{\mathbf{4 0}}\right\}$-modified polyanions [55] (color online). 
$\left\{\mathrm{P}_{2} \mathrm{~W}_{12}\right\}$ 单元三三成组，分别被一个 $\left\{\mathrm{Mn}_{4} \mathrm{~W}_{2}\right\}$ 和两个 $\left\{\mathrm{Mn}_{3} \mathrm{~W}_{3}\right\}$ 单元填补缺位, 通过赤道位的 $\left\{\mathrm{MnO}_{6}\right\}$ 和 $\left\{\mathrm{WO}_{6}\right\}$ 与相邻 $\left\{\mathrm{P}_{2} \mathrm{~W}_{12}\right\}$ 单元连接, 形成 $\left\{\left(\mathrm{Mn}_{4} \mathrm{P}_{2} \mathrm{~W}_{14}\right)-\right.$ $\left.\left(\mathrm{Mn}_{3} \mathrm{P}_{2} \mathrm{~W}_{15}\right)_{2}\right\}$ 单元; $\left\{\mathrm{P}_{8} \mathrm{~W}_{48}\right\}$ 则居于整个阴离子簇的中 心位置, 在结构中起到模板的作用. 值得注意的是, 化 合物合成原料中的 $\mathrm{Mn}$ 以 $\left[\mathrm{Mn}_{12} \mathrm{O}_{12}(\mathrm{OAc})_{16}\left(\mathrm{H}_{2} \mathrm{O}\right)_{4}\right]$ - $4 \mathrm{H}_{2} \mathrm{O} \cdot 2 \mathrm{HOAc}$ 形式存在, 这是首例使用多核 $\mathrm{Mn}$ 簇进行 该类合成的报道. 他们认为, 向外生长的关键在于缓慢 地生成小核的 $\mathrm{Mn}^{\mathrm{III}}$ 中间体, 以阻止与 $\left\{\mathrm{P}_{2} \mathrm{~W}_{12}\right\}$ 单元的快 速反应形成 $\left\{\mathrm{P}_{8} \mathrm{~W}_{48}\right\}$ 竞争产物, 同时还避免了后续歧化 反应中 $\mathrm{Mn}^{\mathrm{II}}$ 阳离子浓度的升高. 时至如今, 以 $\left\{\mathrm{P}_{8} \mathrm{~W}_{48}\right\}$ 作为构筑模块进行多酸合成的研究仍在进行, 例如使 用主族元素对 $\left\{\mathrm{P}_{8} \mathrm{~W}_{48}\right\}$ 单元进行修饰, 由此衍生出 $\left\{\mathrm{Al}_{16}\right\} 、\left\{\mathrm{Se}_{4}\right\}$ 以及有机 As 修饰的 $\left\{\mathrm{As}_{4}\right\}$ 系列化合 物 ${ }^{[57,58,75]}$. 在此类化合物中, 主族金属作为桥联单元修 饰在赤道缺位上，环内的空腔则充斥了大量的碱金属 离子和水分子. 基于大环 $\left\{\mathrm{P}_{8} \mathrm{~W}_{48}\right\}$ 的主客体体系允许构 建构形各异的各种结构，这些结构可能在离子交换、 气体存储、催化等领域显示出有趣的特性.

\section{$3\left\{\mathbf{P}_{2} \mathbf{W}_{12}\right\}$ 基多酸化合物的应用}

在 $\left\{\mathrm{P}_{2} \mathrm{~W}_{12}\right\}$ 多聚体化合物中, 位于聚阴离子外层的 $\left\{\mathrm{P}_{2} \mathrm{~W}_{12}\right\}$ 模块具有优良的得失电子性能, 在多酸骨架中 扮演了储存电子的角色，被修饰于缺位的过渡金属离 子则通常是化合物特殊性质的来源. 当多聚体的修饰 过渡金属为 $\mathrm{Mn} 、 \mathrm{Fe} 、 \mathrm{Co}$ 此类元素时，聚阴离子会根 据过渡金属离子的不同组装方式而表现出各异的磁学 性质. 例如，对于单体 $\left\{\mathrm{Fe}_{9}\right\}$ 和四聚体 $\left\{\mathrm{Fe}_{16}\right\}$ 而言，两例 化合物可以在 $300 \mathrm{~K}$ 下分别呈现出 20 和 $21.9 \mathrm{emu} \mathrm{K} \mathrm{mol}$ 的 $\chi_{\mathrm{m}} \mathrm{T}$ 值, 两者的磁学性能均弱于同数量下的非相互 作用 $\mathrm{Fe}^{\mathrm{III}}$ 中心, 表明这些 $\mathrm{Fe} \mathrm{e}^{\mathrm{III}}$ 中心间存在较强的分子内 反铁磁耦合现象 ${ }^{[32,56]}$. 除可通过结构的设计合成实现 可控调节的磁学性质外, 被 $\mathrm{Nb} 、 \mathrm{Co}$ 修饰的 $\left\{\mathrm{P}_{2} \mathrm{~W}_{12}\right\}$ 多 聚体还能表现出一定的光催化析氢活性，如前文所提 到的化合物 $\left\{\mathrm{Nb}_{\mathbf{1 2}}\right\} 、\left\{\mathrm{Nb}_{\mathbf{3 2}}\right\}^{[60]} 、\left\{\mathrm{Co}_{\mathbf{1 0 . 5}}\right\} 、\left\{\mathrm{Co}_{12}\right\}$ 和 $\left\{\mathbf{C o}_{19}\right\}^{[72]}$, 此方面研究是对 $\left\{\mathrm{P}_{2} \mathrm{~W}_{12}\right\}$ 系列多聚体性能的 有益开拓. 不过, 由于多聚体空腔内用于稳定结构的碱 金属离子, 以 $\left\{\mathrm{P}_{2} \mathrm{~W}_{12}\right\}$ 模块为基础的多聚体结构稳定性 和产率较低, 其相关性能探索往往会受限; 与之相比, $\left\{\mathrm{P}_{8} \mathrm{~W}_{48}\right\}$ 则是 $\left\{\mathrm{P}_{2} \mathrm{~W}_{12}\right\}$ 的自组装产物，可在很大 $\mathrm{pH}$ 范围
(1 8)内的水溶液中保持结构稳定，独特的 $10 \AA$ 直径空 腔与金属离子具有很强的反应性，稳定的结构特征使 $\left\{\mathrm{P}_{8} \mathrm{~W}_{48}\right\}$ 基化合物的应用性质得以开发.

由于POMs的丰富可逆且快速响应的氧化还原行 为, 其大多数化合物可作为均相体系下的电催化剂. 例 如, 在电催化驱动的析氢反应(HER)研究中, Kortz等 ${ }^{[77]}$ 所报道的聚阴离子 $\left[\left\{\mathrm{Co}_{6}\left\{\mathrm{Co}_{9}\left(\beta-\mathrm{SiW}_{8} \mathrm{O}_{31}\right)_{3}\right\}\right]^{5-}\right.$ 和 $\left[\mathrm{Cu}^{\mathrm{II}}{ }_{2-}^{-}\right.$ $\left.\mathrm{Pd}_{22}{ }_{2} \mathrm{P}_{12} \mathrm{O}_{60}(\mathrm{OH})_{8}\right]^{20-}$, Dolbecq等 ${ }^{[8]}$ 报道的POM-MOF化 合物 $\left[\varepsilon-\mathrm{PMo}_{8}{ }_{8} \mathrm{Mo}^{\mathrm{VI}}{ }_{4} \mathrm{O}_{36}(\mathrm{OH})_{4} \mathrm{Zn}_{4}\right]^{[78]}$, 以及经典的Keggin型聚阴离子复合材料均可表现出良好的催化活 性 ${ }^{[79]}$; 而当作为接近应用研究的异相催化剂时, 它们 的水相高溶解度、低表面积等特点却会限制其电活性 的表现. 针对此类问题, 将POM与碳材料、有机聚合 物等材料复合以制备异相催化剂, 或者将POM作为前 驱体合成 $\mathrm{W}_{2} \mathrm{C} 、 \mathrm{Mo}_{2} \mathrm{C}$ 或 $\mathrm{Mo}_{3} \mathrm{~S}_{4}$ 均是可行的解决方 法 ${ }^{[80,81]}$. 例如, 在张光晋等 ${ }^{[82]}$ 的系列研究中, 使用玻碳 板电极对 $\left\{\mathrm{P}_{8} \mathrm{~W}_{48}\right\}$ 溶液进行负电势还原, 可将被还原的 $\mathrm{H}_{16}\left[\mathrm{P}_{8} \mathrm{~W}_{48}\right]$ 与 $\mathrm{rGO}$ 紧密结合, 以形成具有优良HER活性 的纳米复合电催化剂(图10(a)). 实验结果显示, 当电解 体系为 $\mathrm{pH} 1$ 的 $\mathrm{H}_{2} \mathrm{SO}_{4}$ 溶液时, 使用该催化剂在 $100 \mathrm{mV}$ 的过电势下即可达到 $50 \mathrm{~mA} \mathrm{~cm}{ }^{-2}$ 的电流密度, 该性能 甚至优于商业所用的 $\mathrm{Pt} / \mathrm{C}$ 电极; 而在Cadot研究组 ${ }^{[83]}$ 的 研究中, 具有较大空腔的 $\left\{\mathrm{P}_{8} \mathrm{~W}_{48}\right\}$ 模块可作为客体与 $\left[\mathrm{Mo}_{3} \mathrm{~S}_{4}\left(\mathrm{H}_{2} \mathrm{O}\right)_{9}\right]^{4+}$ 共价结合形成化合物 $\left(\mathrm{NBu}_{4}\right)_{14} \mathrm{~K}_{2} \mathrm{H}_{12} \mathrm{P}_{8}-$ $\mathrm{W}_{48}\left(\mathrm{Mo}_{3} \mathrm{~S}_{4}\right)_{3} \mathrm{O}_{184} \cdot 60 \mathrm{H}_{2} \mathrm{O} \quad\left(\left\{\mathbf{M o}_{9} \mathbf{S}_{12}\right\}\right)$, 该化合物可通过 与聚 $(3,4$-乙二氧基塞吩)(PEDOT)复合, 形成适合于光 电析氢反应的复合催化剂(图10(b d )). 此组合方式使 两者间形成了良好的协同效应，这种通过S-POM共价 作用构筑复合物的策略，为复合材料的多尺度构建提 供了一种设计思路.

对于 $\left\{\mathrm{P}_{8} \mathrm{~W}_{48}\right\}^{16-}$ 聚阴离子而言, 团簇的负电荷使其 碱性有所增加, 其还原过程往往伴随着阴离子的质子 化, 因而POM的氧化还原特性对溶液 $\mathrm{pH}$ 值的变化十分 敏感. Ammam研究组 ${ }^{[84]}$ 选用聚丙烯铵盐作为聚电解 质, 通过静电吸附成功实现了与 $\left\{\mathrm{P}_{8} \mathrm{~W}_{48}\right\}^{16-}$ 聚阴离子 的结合, 复合膜与 $\left\{\mathrm{P}_{8} \mathrm{~W}_{48}\right\}$ 的电化学行为相比, 膜内部 酸度的下降导致氧化还原峰向负电势方向偏移, 过程 中峰电流不会随之发生明显变化. 基于以上现象, 通 过比较复合膜修饰电极在待测溶液与标准溶液中的峰 位变化, 即可计算出待测溶液的 $\mathrm{pH}$ 值. 同样, 通过电化 学还原技术可制备出的 $\left\{\mathrm{P}_{8} \mathrm{~W}_{48}\right\}-\mathrm{Au}-\mathrm{rGO}$ 三元复合催 


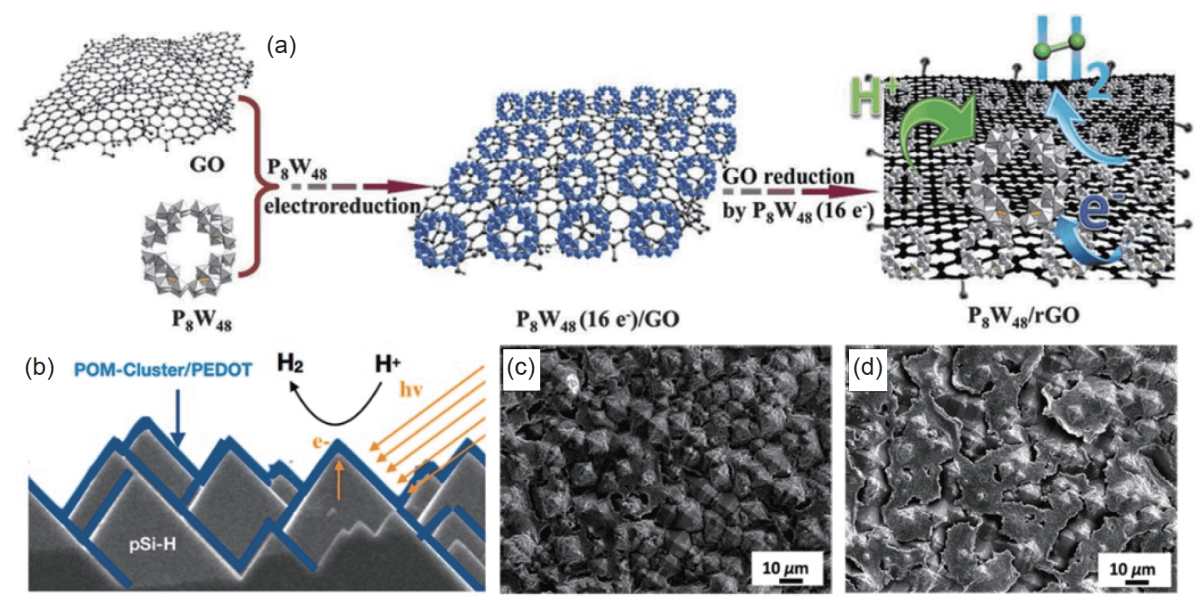

图 10 (a) 复合材料 $\mathrm{P}_{8} \mathrm{~W}_{48} @ \mathrm{rGO}$ 的合成步骤图 ${ }^{[82]}$; (b) $\left\{\mathbf{M o}_{9} \mathrm{~S}_{12}\right\} / \mathrm{PEDOT}$ 光阴极的光致析氢示意图; (c, d) 析氢实验前后, \{Mo $\left.\mathrm{S}_{12}\right\}$ /PEDOT的扫描电子显微镜图 ${ }^{[83]}$ (网络版彩图)

Figure 10 (a) The synthetic steps of $\mathrm{P}_{8} \mathrm{~W}_{48} @ \mathrm{rGO}$ composite [82]; (b) the schematic diagram for hydrogen evolution by $\left\{\mathbf{M o g}_{9} \mathbf{S}_{12}\right\} /$ PEDOT photocathode; and the SEM pictures of $\left\{\mathbf{M o g}_{\mathbf{9}} \mathbf{S}_{12}\right\}$ PEDOT (c) before and (d) after HER experiment [83] (color online).

化剂可用于定量检测 $\mathrm{H}_{2} \mathrm{O}_{2}$, 通过层层自组装法合成的 $\left\{\mathrm{P}_{8} \mathrm{~W}_{48}\right\}-\mathrm{Cu} @ \mathrm{Ag}$ 复合膜也可以对 $\mathrm{IO}_{3}{ }^{-}$表现出良好的电 催化效率(图11(a, b $))^{[85,86]}$. 而在王恩波团队 ${ }^{[37]}$ 对 $\left\{\mathrm{P}_{2} \mathrm{~W}_{12}\right\}$ 二聚体基 $\left\{\mathbf{S n}_{\mathbf{4}} \mathbf{W}\right\}$ 的报道中，该化合物在缓冲 溶液中的循环伏安行为会随着加入 $\mathrm{H}_{2} \mathrm{O}_{2}$ 浓度的不同 发生变化, 即电化学窗口区域电流维持恒定, 电化学还 原区域峰电流整体增大，此现象与 $\left\{\mathrm{P}_{8} \mathrm{~W}_{48}\right\}-\mathrm{Au}-\mathrm{rGO}$ 复 合物表现出的电化学行为一致. 因此可以预计, $\left\{\mathrm{P}_{8} \mathrm{~W}_{48}\right\}^{16-}$ 聚阴离子的这一电化学特性可在其他类似 体系下得到拓展，特别是当前已被证明具有电化学活 性的系列化合物，如已有报道中可对 $\mathrm{NO}_{2}{ }^{-}$表现出电还 原活性的 $\left\{\mathrm{Co}_{6} \mathrm{~W}_{6}\right\} 、\left\{\mathrm{Sb}_{4}\right\} 、\left\{\mathrm{Mn}_{6}\right\}$ 等化合 物 $^{[35,37,64,66,67]}$.

在正常状态下, $\left\{\mathrm{P}_{2} \mathrm{~W}_{12}\right\}$ 多聚体中的 $\mathrm{W}$ 均以 +6 价的 氧化态形式存在，这种状态使多聚体化合物结晶的颜 色更多以修饰金属离子为主; 当多电子转移的发生使 结构中部分 $\mathrm{W}$ 被还原为 +5 价时，还原得到的离域电子 使其产生不同深度的蓝色，这种的混合价态的POM被 称为“杂多蓝”. 由于POM可表现出稳定而可逆的氧化 还原行为, 杂多蓝可以通过电化学方法制备, 稳定可 调的物质颜色使其在光学领域得以应用. 例如, 在刘 绍琴课题组 ${ }^{[87]}$ 的研究中, 可通过 $\left\{\mathrm{P}_{8} \mathrm{~W}_{48}\right\}$ 与 $\mathrm{W}_{18} \mathrm{O}_{49}$ 纳 米线的顺序沉积来制造光调制电致变色设备. 与大多 数报道的设备相比，该复合材料可通过改变施加电势 来选择性地控制近红外和可见光的透射率，进而动态
调控入射光的能量强度, 起到节能环保的作用(图12 (a))；同理，氧化态下的 $\left\{\mathrm{P}_{8} \mathrm{~W}_{48}\right\}$ 仅在紫外光区存在吸 收, 但将其与三邻菲罗啉合钓层间自组装后, 复合膜 会产生一定的电化学诱导荧光行为, 同时苂光行为的 影响程度可通过控制复合膜中 $\left\{\mathrm{P}_{8} \mathrm{~W}_{48}\right\}$ 的层数得以实 现. 在以上工作中, $\left\{\mathrm{P}_{8} \mathrm{~W}_{48}\right\}$ 都作为主要成分对复合物 的光学性能产生了调控作用, 该研究是对POM应用性 质的拓展 ${ }^{[88]}$. 此外, 带有负电荷的 $\left\{\mathrm{P}_{8} \mathrm{~W}_{48}\right\}^{16-}$ 还可以与 聚丙烯酸 $/ \mathrm{ZrO}_{2}$ 结合, 通过改变 $\mathrm{pH}$ 的方式与带正电的蛋 白质产生静电相互作用, 该系列工作已被王建华课题 组报道(图12(b)). 在系列研究中, $\left\{\mathrm{P}_{8} \mathrm{~W}_{48}\right\}$ 表面的 $-\mathrm{OH}$ 基团可与氨基丙基三乙氧基硅烷的 $\mathrm{Si}-\mathrm{OH}$ 发生脱水缩 合, 后通过与聚丙烯酸的缩合形成酰胺键, 此杂化材料 对酸性的卵清蛋白和碱性的溶菌酶表现出了优异的吸 附性能 ${ }^{[89]}$, 两者的吸附容量分别为 367 和 $74 \mathrm{mg} \mathrm{g}^{-1}$; 而 对于 $\left\{\mathrm{P}_{8} \mathrm{~W}_{48}\right\}-\mathrm{ZrO}_{2}$ 复合体系, $\left\{\mathrm{P}_{8} \mathrm{~W}_{48}\right\}$ 组分与糖蛋白羟 基间存在丰富的氢键相互作用，同样使其表现出高度 选择性的糖蛋白分离能力 ${ }^{[90]}$.

\section{4 总结与展望}

随着多酸化学的蓬勃发展, 多酸化合物的相关报 道呈爆发式增长. 本文对近 20 年来过渡金属修饰的 $\left\{\mathrm{P}_{2} \mathrm{~W}_{12}\right\}$ 基多酸化合物的重要成果进行了综述, 主要包 括相关化合物的结构特点、合成方式以及部分报道中 

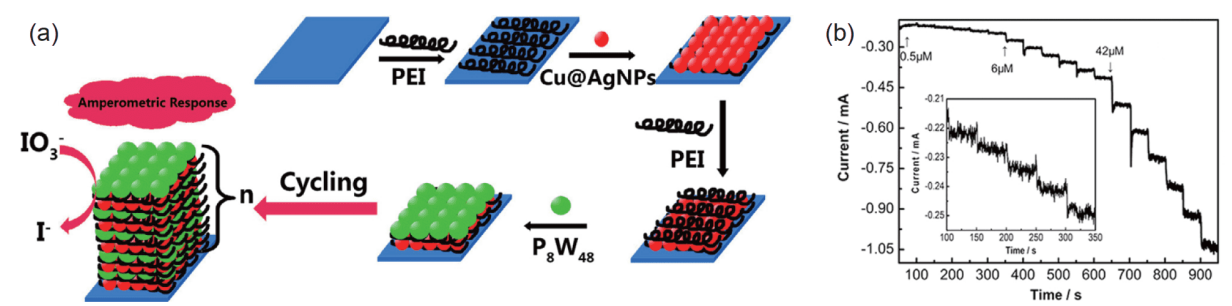

图 11 (a) $\left\{\mathrm{P}_{8} \mathrm{~W}_{48}\right\}-\mathrm{Cu} @ \mathrm{Ag}$ 复合膜的制备示意图 ${ }^{[85]}$; (b) 在改变 $\mathrm{IO}_{3}{ }^{-}$浓度的 $\mathrm{Na}_{2} \mathrm{SO}_{4}-\mathrm{H}_{2} \mathrm{SO}_{4}$ 溶液中的电流-时间响应曲线 ${ }^{[86]}$ (网络 版彩图)

Figure 11 (a) Schematic illustration on the preparation of $\left\{\mathrm{P}_{8} \mathrm{~W}_{48}\right\}-\mathrm{Cu} @ \mathrm{Ag}$ composite film [85], and (b) current-time response curve with varying concentrations of $\mathrm{IO}_{3}{ }^{-}$in $\mathrm{Na}_{2} \mathrm{SO}_{4}-\mathrm{H}_{2} \mathrm{SO}_{4}$ solution [86] (color online).
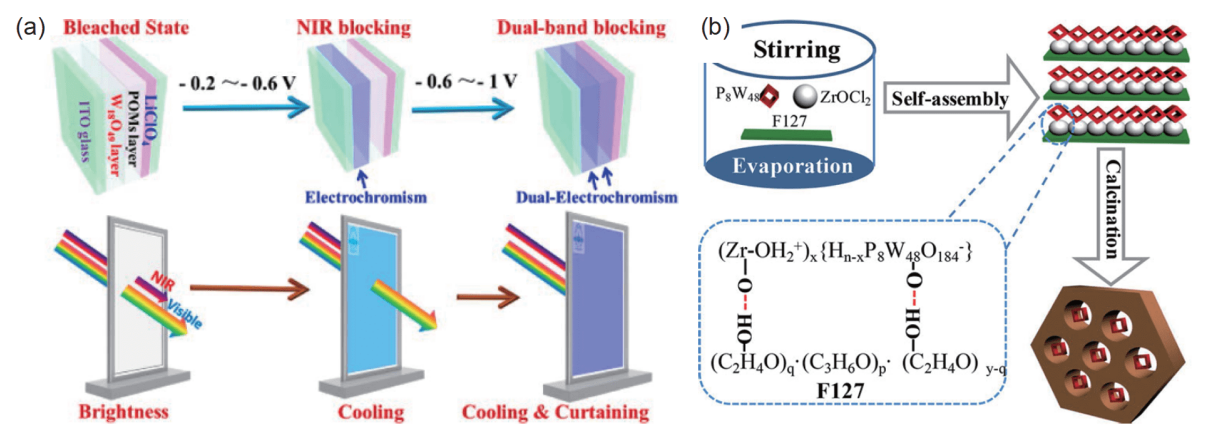

图 12 (a) 基于 $\mathrm{P}_{8} \mathrm{~W}_{48} @ \mathrm{~W}_{18} \mathrm{O}_{49}$ 复合材料对近红外/可见光调制的电致变色器件示意图 ${ }^{[87]}$; (b) 杂化材料 $\left\{\mathrm{P}_{8} \mathrm{~W}_{48}\right\}-\mathrm{ZrO}_{2}$ 的制备过 程示意图 ${ }^{[00]}$ (网络版彩图)

Figure 12 (a) Schematic diagram of $\mathrm{P}_{8} \mathrm{~W}_{48} @ \mathrm{~W}_{18} \mathrm{O}_{49}$ composite-based electrochromic device modulated by near infrared/visible light [87]; (b) schematic illustration on the construction of $\left\{\mathrm{P}_{8} \mathrm{~W}_{48}\right\}-\mathrm{ZrO}_{2}$ hybrid materials [90](color online).

的性能研究. 一系列多聚体的发现和报道丰富了多酸 家族的结构种类与合成策略，为高核团簇的合成提供 了许多有价值的指导. 但迄今为止, 高核多聚体的数 量较少、组装方式略显单一, 已有的最大多聚体仍旧 为2010年所报道的化合物 $\left\{\mathbf{M n}_{40}\right\}$ （该化合物可被看作 $\left\{\mathrm{P}_{2} \mathrm{~W}_{12}\right\}$ 的十六聚体, 或由 12 个 $\left\{\mathrm{P}_{2} \mathrm{~W}_{12}\right\}$ 修饰的 $\left\{\mathrm{P}_{8} \mathrm{~W}_{48}\right\}$ 聚阴离子). 尽管如此, 使用 $\left\{\mathrm{P}_{2} \mathrm{~W}_{12}\right\}$ 模块作为前驱体进 行多聚体的合成仍有迹可循, 同时此类化合物也有很 多潜在性质有待研究与开发. 针对 $\left\{\mathrm{P}_{2} \mathrm{~W}_{12}\right\}$ 多聚体化合 物的合成与性能研究, 我们提出如下潜在的研究方向 与思路.

（1）由于 $\left\{\mathrm{P}_{2} \mathrm{~W}_{12}\right\}$ 在酸性条件下易发生自组装的特 点, 其多聚体相关报道以 $\left\{\mathrm{P}_{8} \mathrm{~W}_{48}\right\}$ 化合物为主, 这一因素 限制了多聚体向更大核数团簇的生长; 但就近年来的报 道而言, $\left\{\mathrm{P}_{8} \mathrm{~W}_{48}\right\}$ 空腔内的活性位点仍旧可以与许多物 质进行共价键合, 形成结构各异的 $\left\{\mathbf{M o}_{8}\right\} 、\left\{\mathbf{M o}_{8} \mathrm{~S}_{8}\right\}$ 、 $\left\{\mathrm{Se}_{4}\right\} 、\left\{\mathrm{Al}_{16}\right\} 、\left\{(\mathrm{PhAs})_{4}\right\}$ 等系列化合物. 从前期的研 究工作可以得出, 多聚体结构中的特殊连接方式来源
于其中具有不同配位能力的异金属离子(如 $\left\{\mathbf{U}_{\mathbf{8}}\right\}$ 、 $\left\{\mathrm{Sb}_{4}\right\} 、\left\{\mathrm{Sn}_{4} \mathrm{~W}\right\} 、\left\{\mathrm{Sn}_{4}\right\}$ 及 $\left\{\mathrm{Nb}_{6}\right\}$ 系列化合物 $)$, 使用离子 半径、成键范围较大的金属离子作为桥联单元，有利 于提升 $\left\{\mathrm{P}_{2} \mathrm{~W}_{12}\right\}$ 前驱体的多聚合组装. 此外, 当使用低 核数过渡金属团簇作为合成原料时，所制备化合物的 结构中往往会存留原料团簇的分解片段, 这些分解片 段对于 $\left\{\mathrm{P}_{2} \mathrm{~W}_{12}\right\}$ 模块的组装具有一定的诱导作用, 有利 于前驱体朝高聚合度的方向组装. 当然, 在使用有机金 属盐作为原料进行合成时，有机部分的碳基组分不参 与配位，可以在组装过程中提供空间位阻; 羧基/羰基 等含氧基团可以与部分过渡金属离子共价连接, 在组 装过程中作为桥联单元; $\left\{\mathrm{P}_{2} \mathrm{~W}_{12}\right\}$ 模块又可以与苯胂 酸/苯膦酸配位结合, 苯基朝聚阴离子的相反方向伸 展. 结合上述三种有机基团与 $\left\{\mathrm{P}_{2} \mathrm{~W}_{12}\right\}$ 模块的相互作 用, 就有望合成 $\left\{\mathrm{P}_{2} \mathrm{~W}_{12}\right\}$ 基POMOF有机-无机杂化物.

(2) 受限于前期工作报道时的研究条件, $\left\{\mathrm{P}_{2} \mathrm{~W}_{12}\right\}$ 多聚体的应用领域集中于磁性和电化学领域. 而在近 年来的研究中, 其应用逐渐拓展到光学材料、光催化 
析氢、吸附材料和质子传输等领域，可见该系列结构 仍旧具有许多未开发的潜在性能. 另外, 在此类研究 中，所涉及材料的成分大多以 $\left\{\mathrm{P}_{8} \mathrm{~W}_{48}\right\}$ 为主，针对其他 $\left\{\mathrm{P}_{2} \mathrm{~W}_{12}\right\}$ 基多聚体的性能开发十分必要．基于 $\mathrm{POM}$ 阴 离子簇的高负电荷数、低比表面积和富氧表面等特 征, 本文涉及的拓展应用均是将 $\left\{\mathrm{P}_{8} \mathrm{~W}_{48}\right\}$ 负载到某种载 体上, 以提高其分散程度和反应活性. 在未来的性能开 发中, 探索合成条件对产物结构的影响, 进而可控修饰 过渡金属离子的类别/数量/空间位置很有必要. 在此基 础上，通过将 $\left\{\mathrm{P}_{2} \mathrm{~W}_{12}\right\}$ 多聚体与金属氧化物/金属纳米
粒子/碳基基底等材料复合，将会是提高其反应活性的 途径之一. 另一方面，鉴于 $\left\{\mathrm{P}_{2} \mathrm{~W}_{12}\right\}$ 多聚体所具有的独 特分子结构，通过使用多孔材料将阴离子簇的位置进 行针定(或通过金属离子/有机配体形成三维网络结 构), 能够形成具有一定体积或特殊形状的空腔, 有望 设计针对特定反应物的限域反应体系. 总之, 尽管目前 已有不少关于此系列化合物的相关报道，结构的可控 合成、产率和稳定性的提升、以及新颖应用领域的拓 展仍旧有较大的研究探索空间, 值得研究者们的关注 并为之付诸努力.

\section{参考文献}

1 Zhang ZM, Zhang T, Wang C, Lin Z, Long LS, Lin W. J Am Chem Soc, 2015, 137: 3197-3200

2 Lv H, Geletii YV, Zhao C, Vickers JW, Zhu G, Luo Z, Song J, Lian T, Musaev DG, Hill CL. Chem Soc Rev, 2012, 41: 7572-7589

3 Lv H, Guo W, Wu K, Chen Z, Bacsa J, Musaev DG, Geletii YV, Lauinger SM, Lian T, Hill CL. J Am Chem Soc, 2014, 136: 14015-14018

4 Wang SS, Yang GY. Chem Rev, 2015, 115: 4893-4962

5 Ma P, Hu F, Wang J, Niu J. Coord Chem Rev, 2019, 378: 281-309

6 Müller A, Peters F, Pope MT, Gatteschi D. Chem Rev, 1998, 98: 239-272

7 Rajaraman G, Murugesu M, Sañudo EC, Soler M, Wernsdorfer W, Helliwell M, Muryn C, Raftery J, Teat SJ, Christou G, Brechin EK. J Am Chem Soc, 2004, 126: 15445-15457

8 Nohra B, El Moll H, Rodriguez Albelo LM, Mialane P, Marrot J, Mellot-Draznieks C, O’Keeffe M, Ngo Biboum R, Lemaire J, Keita B, Nadjo L, Dolbecq A. J Am Chem Soc, 2011, 133: 13363-13374

9 Omwoma S, Gore CT, Ji Y, Hu C, Song YF. Coord Chem Rev, 2015, 286: 17-29

10 Wang D, Liu L, Jiang J, Chen L, Zhao J. Nanoscale, 2020, 12: 5705-5718

11 Bijelic A, Rompel A. Coord Chem Rev, 2015, 299: 22-38

12 Bijelic A, Aureliano M, Rompel A. Angew Chem Int Ed, 2019, 58: 2980-2999

13 Lee SY, Fiene A, Li W, Hanck T, Brylev KA, Fedorov VE, Lecka J, Haider A, Pietzsch HJ, Zimmermann H, Sévigny J, Kortz U, Stephan H, Müller CE. Biochem Pharmacol, 2015, 93: 171-181

14 Liu JC, Han Q, Chen LJ, Zhao JW, Streb C, Song YF. Angew Chem Int Ed, 2018, 57: 8416-8420

Zhao JW, Li YZ, Chen LJ, Yang GY. Chem Commun, 2016, 52: 4418-4445 
Li S, Zhou Y, Ma N, Zhang J, Zheng Z, Streb C, Chen X. Angew Chem Int Ed, 2020, 59: 8537-8540

Kostakis GE, Perlepes SP, Blatov VA, Proserpio DM, Powell AK. Coord Chem Rev, 2012, 256: 1246-1278

Liu ZJ, Wang XL, Qin C, Zhang ZM, Li YG, Chen WL, Wang EB. Coord Chem Rev, 2016, 313: 94-110

Judd DA, Chen Q, Campana CF, Hill CL. J Am Chem Soc, 1997, 119: 5461-5462

Godin B, Chen YG, Vaissermann J, Ruhlmann L, Verdaguer M, Gouzerh P. Angew Chem Int Ed, 2005, 44: 3072-3075

Suzuki K, Minato T, Tominaga N, Okumo I, Yonesato K, Mizuno N, Yamaguchi K. Dalton Trans, 2019, 48: 7281-7289

Godin B, Vaissermann J, Herson P, Ruhlmann L, Verdaguer M, Gouzerh P. Chem Commun, 2005, 5624-5626

Zhang ZM, Yao S, Li YG, Wang YH, Qi YF, Wang EB. Chem Commun, 2008, 1650-1652

Yi X, Izarova NV, Kögerler P. Chem Commun, 2018, 54: 2216-2219

Zhang LC, Zheng SL, Xue H, Zhu ZM, You WS, Li YG, Wang E. Dalton Trans, 2010, 39: 3369-3371

Zhang CD, Liu SX, Ma FJ, Tan RK, Zhang W, Su ZM. Dalton Trans, 2010, 39: 8033-8037

Mal SS, Dickman M, Kortz U. Chem Eur J, 2008, 14: 9851-9855

Mitchell SG, Khanra S, Miras HN, Boyd T, Long DL, Cronin L. Chem Commun, 2009, 2712-2714

Boyd T, Mitchell SG, Miras HN, Long DL, Cronin L. Dalton Trans, 2010, 39: 6460-6465

Assran AS, Izarova NV, Kortz U. CrystEngComm, 2010, 12: 2684-2686

Guo J, Zhao Y, Zhang C, Ma P, Zhang D, Niu J, Wang J. Inorg Chem Commun, 2017, 75: 5-7

Hussain F, Kortz U, Keita B, Nadjo L, Pope MT. Inorg Chem, 2006, 45: 761-766

Minato T, Suzuki K, Yamaguchi K, Mizuno N. Angew Chem Int Ed, 2016, 55: 9630-9633

Zhang D, Cao F, Ma P, Zhang C, Song Y, Liang Z, Hu X, Wang J, Niu J. Chem Eur J, 2015, 21: 17683-17690

Mal SS, Kortz U. Angew Chem Int Ed, 2005, 44: 3777-3780

Pichon C, Mialane P, Dolbecq A, Marrot J, Rivière E, Keita B, Nadjo L, Sécheresse F. Inorg Chem, 2007, 46: 5292-5301

Mitchell SG, Gabb D, Ritchie C, Hazel N, Long DL, Cronin L. CrystEngComm, 2009, 11: 36-39

Müller A, Pope M, Todea A, Bögge H, van Slageren J, Dressel M, Gouzerh P, Thouvenot R, Tsukerblat B, Bell A. Angew Chem Int Ed, 2007, 46: 4477-4480

Sousa FL, Bögge H, Merca A, Gouzerh P, Thouvenot R, Müller A. Chem Commun, 2009, 7491-7493

Korenev VS, Floquet S, Marrot J, Haouas M, Mbomekallé IM, Taulelle F, Sokolov MN, Fedin VP, Cadot E. Inorg Chem, 2012, 51: 2349-2358

Mitchell SG, Streb C, Miras HN, Boyd T, Long DL, Cronin L. Nat Chem, 2010, 2: 308-312

Mitchell SG, Boyd T, Miras HN, Long DL, Cronin L. Inorg Chem, 2011, 50: 136-143

Fang X, Kögerler P, Furukawa Y, Speldrich M, Luban M. Angew Chem Int Ed, 2011, 50: 5212-5216

Mal S, Dickman M, Kortz U, Todea A, Merca A, Bögge H, Glaser T, Müller A, Nellutla S, Kaur N, van Tol J, Dalal N, Keita B, Nadjo L. Chem Eur J, 2008, 14: 1186-1195

Yang P, Alsufyani M, Emwas A-, Chen C, Khashab NM. Angew Chem Int Ed, 2018, 57: 13046-13051

Yi X, Izarova NV, Kögerler P. Inorg Chem, 2017, 56: 13822-13828

Zhang D, Liang Z, Xie S, Ma P, Zhang C, Wang J, Niu J. Inorg Chem, 2014, 53: 9917-9922

Zhang D, Zhang C, Ma P, Bassil BS, Al-Oweini R, Kortz U, Wang J, Niu J. Inorg Chem Front, 2015, 2: 254-262

Zhang D, Luo J, Ma Y, Zhang T, Li N, Li C, Ma P, Li T, Wang G, Liu T, Wang J, Niu J. Inorg Chem, 2020, 59: 6747-6754

Contant R, Abbessi M, Thouvenot R, Hervé G. Inorg Chem, 2004, 43: 3597-3604

Sasaki S, Yonesato K, Mizuno N, Yamaguchi K, Suzuki K. Inorg Chem, 2019, 58: 7722-7729

Yao S, Zhang Z, Li Y, Wang E. Dalton Trans, 2009, 1786-1791

Zhang Z, Yao S, Qi Y, Li Y, Wang Y, Wang E. Dalton Trans, 2008, 3051-3053

Li YW, Li YG, Wang YH, Feng XJ, Lu Y, Wang EB. Inorg Chem, 2009, 48: 6452-6458

Yao S, Zhang Z, Li Y, Wang E. Dalton Trans, 2010, 39: 3884-3889

Yao S, Zhang Z, Li Y, Lu Y, Wang E, Su Z. Cryst Growth Des, 2010, 10: 135-139

Contant R, Teze A. Inorg Chem, 1985, 24: 4610-4614

Liu ZJ, Zhang ZM, Fu H, Li YG, Chen WL, Wu HH, Wang EB. Dalton Trans, 2012, 41: 11700-11705

Ibrahim M, Mbomekallé IM, de Oliveira P, Kostakis GE, Anson CE. Dalton Trans, 2019, 48: 15545-15552 
Jiao YQ, Qin C, Wang XL, Wang CG, Sun CY, Wang HN, Shao KZ, Su ZM. Chem Asian J, 2014, 9: 470-478

Mal SS, Nsouli NH, Dickman MH, Kortz U. Dalton Trans, 2007, 2627-2630

Yi X, Izarova NV, Iftikhar T, van Leusen J, Kögerler P. Inorg Chem, 2019, 58: 9378-9386

Wang KY, Zhang S, Ding D, Ma T, Kortz U, Wang C. Eur J Inorg Chem, 2019, 3-4: 512-516

Zimmermann M, Belai N, Butcher RJ, Pope MT, Chubarova EV, Dickman MH, Kortz U. Inorg Chem, 2007, 46: 1737-1740

Keita B, Kortz U, Holzle LRB, Brown S, Nadjo L. Langmuir, 2007, 23: 9531-9534

Barsukova-Stuckart M, Izarova NV, Jameson GB, Ramachandran V, Wang Z, van Tol J, Dalal NS, Ngo Biboum R, Keita B, Nadjo L, Kortz U. Angew Chem Int Ed, 2011, 50: 2639-2642

Wu HB, Xia BY, Yu L, Yu XY, Lou XWD. Nat Commun, 2015, 6: 6512

Li JS, Wang Y, Liu CH, Li SL, Wang YG, Dong LZ, Dai ZH, Li YF, Lan YQ. Nat Commun, 2016, 7: 11204

Yan G, Wu C, Tan H, Feng X, Yan L, Zang H, Li Y. J Mater Chem A, 2017, 5: 765-772

Liu R, Zhang G, Cao H, Zhang S, Xie Y, Haider A, Kortz U, Chen B, Dalal NS, Zhao Y, Zhi L, Wu CX, Yan LK, Su Z, Keita B. Energy Environ Sci, 2016, 9: 1012-1023

Tourneur J, Fabre B, Loget G, Vacher A, Mériadec C, Ababou-Girard S, Gouttefangeas F, Joanny L, Cadot E, Haouas M, Leclerc-Laronze N, Falaise C, Guillon E. J Am Chem Soc, 2019, 141: 11954-11962

Ammam M, Keita B, Nadjo L, Fransaer J. Sens Actuat B-Chem, 2009, 142: 347-354

Zuo J, Gao N, Yu Z, Kang L, O’Halloran KP, Pang H, Zhang Z, Ma H. J Electroanal Chem, 2015, 751: 111-118

Zhang X, Bao Y, Bai Y, Chen Z, Li J, Feng F. Electrochim Acta, 2019, 300: 380-388

Gu H, Guo C, Zhang S, Bi L, Li T, Sun T, Liu S. ACS Nano, 2018, 12: 559-567

Xu L, Wang B, Gao W, Wu L, Bi L. J Mater Chem C, 2015, 3: 1732-1737

Zhang DD, Guo PF, Hu LL, Chen XW, Wang JH. J Mater Chem B, 2017, 5: 750-756

Zhang D, Wang M, Guo Z, Guo P, Chen X, Wang J. Proteomics, 2018, 18: 1700381

\title{
Advances of transition-metal-modified $\left\{\mathrm{P}_{2} \mathrm{~W}_{12}\right\}$-based polyoxometalates
}

\author{
Xing Xin, Hongjin Lv* \\ MOE Key Laboratory of Cluster Sciences, School of Chemistry and Chemical Engineering, Beijing Institute of Technology, Beijing 102488, China \\ *Corresponding author (email: hlv@bit.edu.cn)
}

\begin{abstract}
Polyoxometalates (POMs) have been emerging as one of the most widely-studied interdisciplinary research fields in Inorganic Chemistry due to their unique structural compositions and physicochemical properties, such as welldefined atomic structure, diverse and controllable composition, stable and reversible electrons/protons storage, rich redox properties. This review addresses the research advances of transition-metal-modified $\left\{\mathrm{P}_{2} \mathrm{~W}_{12}\right\}$-based POMs. These series of compounds have been categorized according to the polymerization number of $\left\{\mathrm{P}_{2} \mathrm{~W}_{12}\right\}$ building block, then the structural characteristics, synthetic strategies, and functional applications of some representative compounds have also been summarized. Finally, the outlook of their future development is prospected.
\end{abstract}

Keywords: polyoxometalates, transition metal modification, $\left\{\mathrm{P}_{2} \mathrm{~W}_{12}\right\}$ and $\left\{\mathrm{P}_{8} \mathrm{~W}_{48}\right\}$ building blocks

doi: $10.1360 /$ SSC-2020-0075 